\title{
Optical observations of planetary nebula candidates from the northern hemisphere ${ }^{\star}$
}

\author{
G.C. Van de Steene ${ }^{1,3, \star \star}$, G.H. Jacoby ${ }^{2}$ and S.R. Pottasch ${ }^{3}$ \\ 1 European Southern Observatory, Casilla 19001, Santiago 19, Chile \\ 2 NOAO , P.O. Box 26732, Tucson, AZ 35726, U.S.A. \\ 3 Kapteyn Astronomical Institute, P.O.Box 800, NL-9700 AV Groningen, The Netherlands
}

Received May 17, 1995; accepted January 8, 1996

\begin{abstract}
We present $\mathrm{H} \alpha+\left[\mathrm{N}_{\mathrm{II}}\right]$ images of 17 and low resolution spectra of 14 IRAS-selected planetary nebula candidates. The $\mathrm{H} \alpha+\left[\mathrm{N}_{\mathrm{II}}\right]$ images are presented as finding charts. Contour plots are shown for the resolved planetary nebulae. From these images accurate optical positions and mean optical angular diameters were determined. Optical spectra show that the IRAS-selected and radio detected planetary nebula candidates are indeed planetary nebulae. Three planetary nebula candidates, previously not detected in the radio continuum were seen in H $\alpha$. They are larger, low surface brightness planetary nebulae. Most of these IRAS planetary nebulae are heavily extinct, having an average $A_{V}$ of 7 magnitudes. About half of the planetary nebulae seem to be of low excitation, having central stars with an effective temperature probably $\sim 60.000 \mathrm{~K}$ or less.
\end{abstract}

Key words: planetary nebulae: general

\section{Introduction}

This is a continuation of the project to identify new Planetary Nebulae (PN) applying the method as proposed by Pottasch et al. (1988). Unidentified IRAS sources were selected from the IRAS Point Source Catalog (PSC) on the basis of far IR colors, which are typical of PN. Having selected the candidates, there are two steps in proving that they are PN: the first is to observe these objects in the radio continuum, and the second is to take optical spectra. Radio measurements are especially suitable for a first confirmation, because in this wavelength range the radiation is not attenuated by extinction. Additionally, the detection of radio continuum emission shows the presence of ionized gas, which is strong evidence that the object is a PN. Finally, with radio synthesis observations one can obtain accurate positions to verify a correct association and which are useful for optical identification. This was successfully done for PN candidates inside the galactic bulge (Pottasch et al. 1988; Ratag et al. 1990; Ratag \& Pottasch 1991) and outside the galactic bulge (Van de

Send offprint requests to: G.C. Van de Steene ${ }^{1}$

${ }^{\star}$ Based on data acquired at Kitt Peak National Observatory

${ }^{\star \star}$ Visiting Astronomer at Kitt Peak National Observatory, National Optical Observatories, which is operated by the Association of Universities for Research in Astronomy, Inc., under cooperatieve agreement with the National Science Foundation
Steene \& Pottasch 1993, 1995). Second, to confirm that these IRAS-selected and radio detected PN candidates are indeed PN, they should be observed in the optical.

In this paper we present optical images of 17 and spectroscopy of 14 PN candidates, observable from the northern hemisphere (Van de Steene \& Pottasch 1995; hereafter Paper I).

\section{Selection of the planetary nebula candidates}

The sample candidates were selected from the IRAS PSC on the basis of their colors that are typical of PN. Only IRAS sources falling within the color box $F 12 / F 25 \leq 0.35$ and $F 25 / F 60 \geq 0.35$ were chosen, to avoid confusion with galaxies and HII regions, having colors just outside this box $(F 12, F 25$, and $F 60$ refer to the flux in the IRAS wavelength bands centered at 12,25 and $60 \mu \mathrm{m}$ respectively). About half the sample sources have good flux values (quality 2 or 3 ) at 12,25 , and $60 \mu \mathrm{m}$ and the other half have an upper-limit at $12 \mu \mathrm{m}$, but very good quality (3) flux values at 25 and $60 \mu \mathrm{m}$. The PN candidates are further than 15 degrees in longitude from the galactic center, presumably outside the galactic bulge The list of these IRAS-selected and radio observed PN candidates was presented in Paper I. 


\section{Observations}

\subsection{Imaging}

Images of all IRAS-selected PN candidates were taken through an $\mathrm{H} \alpha+[\mathrm{N}$ II] filter with the KPNO $0.9 \mathrm{~m}$ and 4 $\mathrm{m}$ telescopes at Kitt Peak National Observatory (KPNO), USA, during 1992 and 1993. The main goal of taking the $\mathrm{H} \alpha$ images was to identify the $\mathrm{PN}$ candidates, then to obtain accurate optical positions and use the images as finding charts for optical spectroscopy. We also wanted to determine the mean optical angular diameters of the PN, in order to compare them with those obtained from the radio continuum images.

Table 1. Imaging observing log of detected PN candidates

\begin{tabular}{lllll}
\hline Object & Observers & Date & Tel. & Time \\
\hline $1818-0833$ & Jacoby/Ciardullo & $05 / 04 / 92$ & $4 \mathrm{~m}$ & $120 \mathrm{~s}$ \\
$1823-1047$ & Jacoby/McMillan & $19 / 04 / 93$ & $4 \mathrm{~m}$ & $300 \mathrm{~s}$ \\
$1824-1410$ & Jacoby/Ciardullo & $05 / 04 / 92$ & $4 \mathrm{~m}$ & $120 \mathrm{~s}$ \\
$1825-0940$ & Jacoby/Ciardullo & $05 / 04 / 92$ & $4 \mathrm{~m}$ & $120 \mathrm{~s}$ \\
$1827-0729$ & Jacoby/McMillan & $19 / 04 / 93$ & $4 \mathrm{~m}$ & $300 \mathrm{~s}$ \\
$1839-1418$ & Jacoby/McMillan & $19 / 04 / 93$ & $4 \mathrm{~m}$ & $200 \mathrm{~s}$ \\
$1840-1109$ & Jacoby/Ciardullo & $06 / 04 / 92$ & $4 \mathrm{~m}$ & $120 \mathrm{~s}$ \\
$1841+0343$ & VdSteene/Jacoby & $24 / 05 / 92$ & $0.9 \mathrm{~m}$ & $600 \mathrm{~s}$ \\
$1858+0821$ & Jacoby/McMillan & $19 / 04 / 93$ & $4 \mathrm{~m}$ & $200 \mathrm{~s}$ \\
$1859+1013$ & Jacoby/Ciardullo & $06 / 04 / 92$ & $4 \mathrm{~m}$ & $120 \mathrm{~s}$ \\
$1904+1038$ & VdSteene/Jacoby & $11 / 06 / 93$ & $0.9 \mathrm{~m}$ & $600 \mathrm{~s}$ \\
$1908+0422$ & VdSteene/Jacoby & $11 / 06 / 93$ & $0.9 \mathrm{~m}$ & $600 \mathrm{~s}$ \\
$1909+1326$ & VdSteene/Jacoby & $25 / 05 / 92$ & $0.9 \mathrm{~m}$ & $600 \mathrm{~s}$ \\
$1911+1534$ & VdSteene/Jacoby & $25 / 05 / 92$ & $0.9 \mathrm{~m}$ & $600 \mathrm{~s}$ \\
$1920+1122$ & VdSteene/Jacoby & $11 / 06 / 93$ & $0.9 \mathrm{~m}$ & $600 \mathrm{~s}$ \\
$1933-0400$ & VdSteene/Jacoby & $14 / 06 / 93$ & $0.9 \mathrm{~m}$ & $60 \mathrm{~s}$ \\
$1943+2251$ & VdSteene/Jacoby & $11 / 06 / 93$ & $0.9 \mathrm{~m}$ & $600 \mathrm{~s}$ \\
\hline
\end{tabular}

Some images were taken with the KPNO $0.9 \mathrm{~m}$ telescope using the T2KA CCD during May 1992 and June 1993. The PN candidates were observed through the $\mathrm{H} \alpha+\left[\mathrm{N}_{\mathrm{II}}\right]$ filter KPNO-1276 with a FWHM of $35 \AA$ centered at $6565 \AA$ and in the continuum through the KPNO1376 filter with a FWHM of $271 \AA$ centered at $6098 \AA$. The pixel size of the images taken with the KPNO $0.9 \mathrm{~m}$ telescope is 0 .' 68 per pixel.

Some images were taken with the KPNO $4 \mathrm{~m}$ telescope using the T1KA CCD in 1992 and the T2KB CCD in 1993. The PN candidates were observed through the $\mathrm{H} \alpha+[\mathrm{N}$ II] filter KPNO-1390 with a FWHM of $72 \AA$ centered at $6587 \AA$. The continuum images were taken through the Harris $R$ filter. The pixel size of the images taken with KPNO $4 \mathrm{~m}$ telescope is 0 "' 47 per pixel.

The images were reduced using standard procedures in the IRAF reduction package as described by Massey (1992). Afterwards the images were searched for the presence of a PN near the center of the field using the following procedure: each $\mathrm{H} \alpha+\left[\mathrm{N}_{\mathrm{II}}\right]$ image was divided by the continuum one, and all three images were blinked.
Table 1 gives the observation log for the $\mathrm{H} \alpha$ images in which a PN was identified. The first column indicates the $\mathrm{PN}$, the second column the observer, the third column the observing date, the fourth column the telescope used and the fifth the exposure time of the images.

\subsection{Spectroscopy}

The $\mathrm{H} \alpha$ images were used as finding charts for spectroscopy. Spectra were taken at the KPNO $2.1 \mathrm{~m}$ telescope with Goldcam in July 1993. The slit aperture was $2 . ! 5$, the length was 2.5 and the orientation east-west. The spectral range is from $3,600 \AA$ to $7,400 \AA$ and the spectral resolution is $\sim 8 \AA$.

We first positioned a bright, nearby HST guide star in the slit and then offset the telescope to the accurately determined optical position of the PN. We used this offset process because these PN generally are not visible on an acquisition TV. Using the image we ascertained that we had the right object in the slit. The weather was not photometric. Due to cirrus we lost a variable amount of photons, even when the guide star remained visible. Sometimes the exposure had to be stopped. The PN candidate, the date and the actual exposure time are listed in Table 2 .

Table 2. Spectroscopy observing log

\begin{tabular}{lll}
\hline Object & Date & Time \\
\hline $1818-0833$ & $17 / 07 / 93$ & $30 \mathrm{~min}$ \\
$1823-1047$ & $20 / 07 / 93$ & $30 \mathrm{~min}$ \\
$1824-1410$ & $19 / 07 / 93$ & $30 \mathrm{~min}$ \\
$1825-0940$ & $19 / 07 / 93$ & $45 \mathrm{~min}$ \\
$1827-0729$ & $18 / 07 / 93$ & $30 \mathrm{~min}$ \\
$1839-1418$ & $18 / 07 / 93$ & $25 \mathrm{~min}$ \\
$1840-1109$ & $17 / 07 / 93$ & $10 \mathrm{~min}$ \\
$1841+0343$ & $18 / 07 / 93$ & $30 \mathrm{~min}$ \\
$1858+0821$ & $17 / 07 / 93$ & $15 \mathrm{~min}$ \\
$1859+1013$ & $17 / 07 / 93$ & $15 \mathrm{~min}$ \\
$1908+0422$ & $16 / 07 / 93$ & $54 \mathrm{~min}$ \\
$1909+1326$ & $18 / 07 / 93$ & $45 \mathrm{~min}$ \\
$1911+1534$ & $19 / 07 / 93$ & $45 \mathrm{~min}$ \\
$1920+1122$ & $20 / 07 / 93$ & $45 \mathrm{~min}$ \\
$1933-0400$ & $19 / 07 / 93$ & $30 \mathrm{~min}$ \\
$1943+2251$ & $18 / 07 / 93$ & $45 \mathrm{~min}$ \\
\hline
\end{tabular}

The spectra were reduced using standard procedures in IRAF, as described by Massey et al. (1992). The wavelength calibration was done using a HeNeAr calibration lamp. For deriving the response curve of the detector, 3 to 4 standard stars were observed each night, except for the first night, when the weather was so bad that we only observed one standard star. 
Table 3. Comparison between radio and optical positions. All positions are given in 1950.0 coordinates

\begin{tabular}{|c|c|c|c|c|c|c|c|c|}
\hline Object & $l$ & $b$ & $\begin{array}{l}\text { RA } \\
\text { IRAS } \\
\text { h m s }\end{array}$ & $\begin{array}{l}\text { DEC } \\
\text { IRAS } \\
\circ, \| \prime\end{array}$ & $\begin{array}{l}\text { RA } \\
\text { Radio } \\
\text { h m s }\end{array}$ & $\begin{array}{l}\text { DEC } \\
\text { Radio } \\
0,11\end{array}$ & $\begin{array}{l}\text { RA } \\
\text { Optical } \\
\text { h m s }\end{array}$ & $\begin{array}{l}\text { DEC } \\
\text { Optical } \\
\circ, \prime \prime\end{array}$ \\
\hline$\overline{1818-0833}$ & 21.9 & 2.7 & 181836.8 & -083312 & 181837.3 & -083309 & - & - \\
\hline $1823-1047$ & 20.4 & 0.6 & 182311.8 & -104715 & 182311.6 & -104716 & 182311.8 & -104715 \\
\hline $1824-1410$ & 17.6 & -1.2 & 182421.3 & -141036 & 182422.8 & -141027 & 182422.8 & -141029 \\
\hline $1825-0940$ & 21.6 & 0.8 & 182501.1 & -094008 & 182500.6 & -094009 & 182500.7 & -094007 \\
\hline $1827-0729^{i}$ & 23.9 & 1.2 & 182748.9 & -072945 & 182747.9 & -072945 & 182748.0 & -072945 \\
\hline $1840-1109^{i i}$ & 22.0 & -3.1 & $\begin{array}{lll}18 & 40 & 10.4\end{array}$ & -110956 & $\begin{array}{lll}18 & 40 & 10.3\end{array}$ & -110956 & - & - \\
\hline $1858+0821$ & 41.5 & 1.7 & 185841.5 & 082116 & 185841.5 & 082120 & 185841.6 & 082117 \\
\hline $1904+1038$ & 44.1 & 1.5 & 190410.7 & 103841 & 190410.4 & 103841 & 190410.6 & 103841 \\
\hline $1908+0422$ & 39.1 & -2.2 & 190824.1 & 042226 & 190824.4 & 042228 & 190824.5 & 042228 \\
\hline $1909+1326$ & 47.2 & 1.8 & 190917.6 & 132606 & 190917.3 & 132609 & 190917.4 & 132608 \\
\hline $1911+1534$ & 49.3 & 2.4 & 191102.9 & 153427 & 191103.1 & 153431 & 191103.2 & 153430 \\
\hline $1920+1122$ & 46.7 & -1.6 & 192029.0 & 112203 & 192029.1 & 112209 & 192029.3 & 112206 \\
\hline $1933-0400$ & 34.5 & -11.7 & 193339.5 & -040008 & 193339.5 & -040000 & 193339.6 & -040008 \\
\hline $1943+2251$ & 59.4 & -0.7 & 194326.1 & 225111 & 194325.8 & 225112 & 194325.9 & 225112 \\
\hline$\overline{1839-1418}$ & 19.2 & -4.5 & 183933.9 & -141811 & 183934.4 & -141810 & - & \\
\hline $1841+0343$ & 35.4 & 3.5 & 184107.0 & 034335 & $1841 \quad 07.2$ & 034337.1 & - & \\
\hline $18599+1013^{i i i}$ & 43.3 & 2.2 & 185956.1 & 101308 & 185956.1 & 101312 & - & \\
\hline $\begin{array}{cc}i & \text { PN G 2 } \\
i i & \text { PN G } \\
i i i & \text { PN G }\end{array}$ & $\begin{array}{l}3.1 \\
-2.2\end{array}$ & hal & $\begin{array}{l}\text { 1992) } \\
\text { ki 1946) }\end{array}$ & & & & & \\
\hline
\end{tabular}

\section{Discussion}

\subsection{Imaging}

The images of all identified PN candidates are presented in Appendix A, as finding charts. If the PN was resolved, its contour plot is shown as well in Appendix B.

Table 4. Comparison between radio and optical angular diameters. All angular sizes are given in arcseconds

\begin{tabular}{lrrrr}
\hline Object & $\theta_{\mathrm{PSF}}$ & $\theta_{\text {FWHM }}$ & $\theta_{\text {Str }}$ & $\theta_{\text {Str }}$ \\
& $\mathrm{H} \alpha$ & $\mathrm{H} \alpha$ & $\mathrm{H} \alpha$ & $6 \mathrm{~cm}$ \\
\hline $1818-0833$ & 1.5 & 1.7 & 3.1 & 6.8 \\
$1823-1047$ & 2.1 & 2.5 & 4.5 & 6.6 \\
$1824-1410$ & 1.7 & ext. & 11.2 & 17.8 \\
$1825-0940$ & 2.3 & 2.5 & 4.5 & 3.6 \\
$1827-0729^{i}$ & 2.9 & $<2.9$ & $<4.7$ & 7.1 \\
$1840-1109^{i i}$ & 1.7 & ext. & 11.7 & 12.8 \\
$1858+0821$ & 1.5 & 1.0 & 1.8 & $<13$ \\
$1904+1038$ & 2.0 & 3.2 & 5.8 & 6.4 \\
$1908+0422$ & 1.6 & ext. & 10.7 & $<17$ \\
$1909+1326$ & 1.3 & $<1.3$ & $<2.3$ & $<3.1$ \\
$1911+1534$ & 1.4 & $<1.4$ & $<2.4$ & $<4.1$ \\
$1920+1122$ & 2.1 & ext. & 6.9 & $<8.2$ \\
$1933-0400$ & 1.8 & 0.8 & 1.4 & $<5.7$ \\
$1943+2251$ & 1.4 & 2.2 & 4.0 & $<7.7$ \\
\hline $1839-1418$ & 1.9 & ext. & 17.5 & - \\
$1841+0343$ & 1.6 & ext. & $4.5 \times 13.8$ & - \\
$1859+1013$ & 2.0 & ext. & 17.9 & - \\
\hline
\end{tabular}

\subsubsection{Positional accuracy}

The accurate optical position of the PN was obtained using the HST guide star catalog and the package FINDER in IRAF. The PN, its galactic longitude and latitude, the radio position and the optical position in 1950.0 coordinates are listed in Table 3.

For almost all PN, the optical position is within $3^{\prime \prime}$ of the radio position. The accuracy of the optical positions is similar to the positional uncertainty of the HST guide stars, and thus better than $1^{\prime \prime}$ in right ascension (RA) and declination (DEC). The accuracy of the radio positions depends upon the beamsize and the signal-to-noise ratio. The RA, obtained from the radio measurement, is also expected to be better than $1^{\prime \prime}$. The DEC, obtained from the radio measurements, also depends upon $\sin (\mathrm{DEC})$. Because our sample $\mathrm{PN}$ are at low declinations, the accuracy in DEC could be worse than $1^{\prime \prime}$. Therefore the optical declination is generally expected to be the most accurate.

For PN 1818-0833 and 1840-1109 insufficient HST guide stars were present in the field imaged to obtain an optical position. For the PN 1839-1418, 1841+0343 and $1859+1013$, detected in $\mathrm{H} \alpha$ but not in the radio, the IRAS position is given.

The optical declination of PN 1933-0400 is $8^{\prime \prime}$ off in declination compared to the radio position, but exactly on the IRAS position. Because the uncertainty of the declination in the radio is estimated to be $8^{\prime \prime}\left( \pm 5^{\prime \prime}\right)$, the optical declination is within the accuracy of the radio 
position. Therefore the radio, optical and IRAS sources are considered associated.

The optical PN $1859+1013$ is about ten times the maximum uncertainty in declination away from the radio position: $\mathrm{RA}=18^{\mathrm{h}} 59^{\mathrm{m}} 55^{\mathrm{s}} .4$ and $\mathrm{DEC}=10^{\circ} 14^{\prime} 00^{\prime \prime}$. However the IRAS position is in perfect agreement with the optical position. Consequently, the radio source is probably not associated with the optical PN. Another indication is that, based on its $\mathrm{H} \beta$ flux, PN 1859+1013 would have a radio flux below 1 mJy per beam, which is much below our detection limit of $\sim 3 \mathrm{mJy} /$ beam.

The optical position of PN $1841+0343$ is in perfect agreement with the IRAS position. In the radio map centered on the IRAS position, two radio sources are present: one of $7.1 \mathrm{mJy}$ at $\mathrm{RA}=18^{\mathrm{h}} 41^{\mathrm{m}} 09^{\prime \prime} \cdot 7$ and $\mathrm{DEC}=3^{\circ} 44^{\prime} 46^{\prime \prime}$ mJy, and one of $4.0 \mathrm{mJy}$ at $\mathrm{RA}=18^{\mathrm{h}} 41^{\mathrm{m}} 07^{\mathrm{s}} .1$ and $\mathrm{DEC}=$ $3^{\circ} 44^{\prime} 42^{\prime \prime}$. The first one is definitely too far away to be associated with the IRAS position. The second one is about three times further off the IRAS source in declination than its positional uncertainty. We don't think that any of the radio sources is associated with the $\mathrm{PN}$. This needs to be confirmed.

\subsubsection{Angular diameters}

Optical: The point-spread function is well approximated by a gaussian with a FWHM of $\theta_{\mathrm{PSF}}$, derived from the second moments of the profiles of field stars and given in Col. 2 of Table 4. We also determined in this way the FWHM of the PN itself, $\theta_{\mathrm{PN}}$, and then calculated its deconvolved FWHM according to $\theta_{\mathrm{FWHM}}=\sqrt{\theta_{\mathrm{PN}}^{2}-\theta_{\mathrm{PSF}}^{2}}$ (given in Col. 3 of Table 4). However a gaussian profile is a rather poor description of the intensity distribution of a PN. In the case of a partially resolved object the best approach is to calculate $\theta_{\mathrm{FWHM}}$ and then apply a correction factor based on an assumed intensity distribution to obtain the true Strömgren angular diameter of the PN (Panagia \& Walmsley 1978; Bedding \& Zijlstra 1994). The Strömgren angular diameters, $\theta_{\mathrm{Str}}$, (given in Cols. 4 and 5 of Table 4) were calculated assuming that the ratio of the inner radius to the Strömgren radius is equal to a half. From the resulting intenstity distribution we determined that $\theta_{\text {FWHM }}$ should be multiplied with a correction factor of 1.8 to obtain the Strömgren radius (van Hoof 1996, in preparation).

The PN for which the gaussian diameter is stellar an upper limit is given. The angular sizes of the well resolved $\mathrm{PN}$ were measured in RA and DEC from the lowest contour in the plots presented in Appendix B. Then the geometric mean diameter was calculated and listed as $\theta_{\text {Str }}$ in Table 4 .

Radio: For comparison the radio diameters are given in Table 4 . The radio angular diameters are likely to be overestimated, due to the very elongated beam compared to the small sizes of the PN, and the lack of $u v$-coverage. From Table 4 it is clear that most radio diameters are larger than the optical diameters, and that the difference is worse than for the PN observed with the Australian Compact Array (Van de Steene \& Pottasch 1993).

\subsubsection{Detection statistics}

Table 5 gives an overview of the IRAS PN candidates which were observed in the radio continuum, those which were detected (det), and those which were observed (obs) in the optical.

The radio detected candidate $1903+0801$ (Paper I) wasn't observed in the optical.

The following PN candidates were detected in the radio continuum but not in $\mathrm{H} \alpha$ : $1853+0549,1917+1252$, $1935+2302$. If they are indeed PN, they are probably undetected because of extinction (Kistiakowsky \& Helfland 1993). The region around PN $1853+0549$ is clearly very obscured.

The only PN detected in $\mathrm{H} \alpha$ for which we didn't obtain a spectrum is $1904+1038$. We obtained spectra of PN 18250940 and PN 1858+0821, but the signal-to-noise ratios were so low, that we can't confirm their PN nature.

Table 5. Overview: detections and non-detections

\begin{tabular}{llll}
\hline Object & $\mathrm{S}_{6 \mathrm{~cm}}$ & $\mathrm{H} \alpha$ & Spectrum \\
\hline $1818-0833$ & det & det & det \\
$1824-1410$ & det & det & det \\
$1825-0940$ & det & det & too low S/N \\
$1840-1109$ & det & det & det \\
$1909+1326$ & det & det & det \\
$1911+1534$ & det & det & det \\
$1917+1252$ & det & not det & not obs \\
\hline $1823-1047$ & det & det & det \\
$1827-0729$ & det & det & det \\
$1853+0549$ & det & not det & not obs \\
$1858+0821$ & det & det & too low S/N \\
$1903+0801$ & det & not obs & not obs \\
$1904+1038$ & det & det & not obs \\
$1908+0422$ & det & det & det \\
$1920+1122$ & det & det & det \\
$1933-0400$ & det & det & det \\
$1935+2302$ & det & not det & not obs \\
$1943+2251$ & det & det & det \\
\hline $1839-1418$ & not det & det & det \\
$1841+0343$ & not det & det & det \\
$1859+1013$ & not det & det & det \\
\hline
\end{tabular}

We obtained $\mathrm{H} \alpha+[\mathrm{NII}]$ images of all $\mathrm{PN}$ candidates from Paper I which have a RA larger than $18^{\mathrm{h}}$, thus also of the forty candidates which weren't detected in the radio continuum. The larger PN: 1839-1418, 1841+0343, and $1859+1013$, were detected in the $\mathrm{H} \alpha$ images, but not in our radio continuum observations. Based on their observed $\mathrm{H} \beta$ fluxes PN 1839-1418, 1841+0343, and 1859+1013 would 
have a radio flux below $1 \mathrm{mJy}$ per beam, which is much below the detection limit of $\sim 3 \mathrm{mJy} /$ beam.

\subsection{Spectroscopy}

The spectra of the PN candidates detected in $\mathrm{H} \alpha$ are shown in Appendix C. The Table with line-identifications and line-ratios normalized to $\mathrm{H} \beta$, or $[\mathrm{O} \mathrm{III}] \lambda 5007$, depending upon which one was available and best determined, are presented in Appendix D. The line-fluxes were corrected for interstellar extinction using the extinction law from Seaton (1979). This typical interstellar extinction law and the observed $\mathrm{H} \alpha / \mathrm{H} \beta$ ratio, when compared to the recombination value of 2.85 (Aller 1984), gives a logarithmic extinction at $\mathrm{H} \beta$ :

Table 6. Values for logarithmic extinction, $A_{V}(\mathrm{mag})=2.1$ $c(\mathrm{H} \beta)=3.1 c(\mathrm{H} \alpha)$

\begin{tabular}{lrrrr}
\hline Object & $c(\mathrm{H} \beta)$ & $c(\mathrm{H} \alpha)$ & $\begin{array}{r}A_{V} \\
\mathrm{mag}\end{array}$ & $\begin{array}{r}\text { Excitation } \\
\text { Class }\end{array}$ \\
\hline $1818-0833$ & 2.7 & & 5.7 & 5.5 \\
$1823-1047$ & 5.3 & & 11.1 & 4.0 \\
$1824-1410$ & 3.3 & & 6.9 & 5.1 \\
$1825-0940$ & - & 2.1 & 6.5 & - \\
$1827-0729$ & 4.6 & & 9.7 & 0.1 \\
$1839-1418$ & 1.5 & & 3.2 & 9.8 \\
$1840-1109$ & 1.1 & & 2.3 & 7.3 \\
$1841+0343$ & 2.3 & & 4.8 & 0.3 \\
$1858+0821$ & - & 3.4 & 10.5 & - \\
$1859+1013$ & 1.9 & & 4.0 & 4.0 \\
$1908+0422$ & - & 2.8 & 8.7 & 8.0 \\
$1909+1326$ & 3.2 & & 8.7 & 7.7 \\
$1911+1534$ & - & 2.7 & 8.4 & 5.7 \\
$1920+1122$ & - & 2.8 & 8.7 & 8.4 \\
$1933-0400$ & 0.8 & & 1.7 & 0.0 \\
$1943+2251$ & - & 3.0 & 6.3 & 11.0 \\
\hline
\end{tabular}

$c(\mathrm{H} \beta)=3.096 \log (\mathrm{H} \alpha /(\mathrm{H} \beta 2.85)$,

which corresponds to a visual extinction of $A_{V}=2.1 c(\mathrm{H} \beta)$. For PN without detectable $\mathrm{H} \beta$ emission, we used the previous expression for $c(\mathrm{H} \beta)$, together with the equation by Pottasch (1984) based on the radio flux:

$c(\mathrm{H} \beta)=\log \left(S_{6 \mathrm{~cm}} /(3.67 \mathrm{H} \beta)\right.$,

to eliminate $\mathrm{H} \beta$ from the equations and to derive the logarithmic extinction value at $\mathrm{H} \alpha$ (i.e. $c(\mathrm{H} \alpha)$ ), which corresponds to a visual extinction of $A_{V}=3.1 c(\mathrm{H} \alpha)$. However this is a rough estimate, because for the larger nebulae, some flux will have been missed due to the $2^{\prime \prime}$ slit width, while the radio flux is measured over the whole PN. Furthermore the weather wasn't photometric, so that standard stars and PN weren't observed under the same sky conditions. The values for extinction based on $\mathrm{H} \alpha$ and ra- dio flux are therefore likely to be less accurate. The values for the logarithmic extinctions are presented in Table 6 . The fact that no $\mathrm{H} \beta$ emission was seen, is in itself a clear indication for a high extinction with $A_{V}$ larger than $\sim 8$ mag. While the values for $A_{V}$ of optically known PN are in the range 0.04 to $4.8 \mathrm{mag}$ (Osterbrock 1989), we notice that for only 5 of these 16 IRAS PN, $A_{V}$ is smaller than 4.8. These IRAS PN have an average $A_{V}=6.7 \mathrm{mag}$.

After correction for extinction, the excitation class of the PN was calculated according to Dopita \& Meatheringham (1990). The excitation classes are given in Col. 5 of Table 6 . The relation between excitation class and stellar effective temperature is rather analogous to the relation between stellar spectral type and effective temperature in the case of stars. An excitation class higher than 5 means that He II $\lambda 4686$ could be observed in the spectrum and that the stellar temperature is higher $\sim 60,000 \mathrm{~K}$. From Table 6 we see that about half the number of PN are of low excitation.

\section{Notes on individual objects}

We searched the literature using the Simbad database to collect published information about the individual PN. All PN candidates except PN 1824-1410 were listed in PreiteMartinez (1988) as possible new PN. Next we checked whether the IRAS sources had an association in the "Catalogue of galactic PN" (Acker et al. 1992).

$1818-0833$

PK $021+02.1$ was listed as a suspected PN by MacConnel (1978) and associated with the IRAS source 18186-0833 by Iyengar (1987). Manchado et al. (1989) in an abstract in the Revista Mexicana, mentioned that from low resolution spectra they found the object to be a PN of low excitation, which seems to have a high helium abundance. The nebula has no He II $\lambda 4686$ emission, indicative of a stellar temperature below $\sim 60,000 \mathrm{~K}$.

\section{$1823-1047$}

If we compare the spectrum of this $\mathrm{PN}$ to the one above they seem quite similar, despite the much higher extinction of PN 1823-1047.

$1824-1410$

Some interstellar $\mathrm{H} \alpha$ emission of material further away from the brighter PN is visible in the image, and it is not clear whether it has been associated with the PN. If one forget about the foreground stars, the contour plot of this PN looks much like PN 1908+0422, only the center seems brighter.

\section{$1825-0940$}

The spectrum of this probable PN has a very low signal to noise and only $\mathrm{H} \alpha,[\mathrm{N} \mathrm{II}] \lambda 6548 \& 6583,[\mathrm{Ar}$ III $] \lambda 7135$ and $[\mathrm{O}$ II $] \lambda 7323 \& 7332$ are observed. Their line strengths after correction for extinction to $\mathrm{H} \alpha=100$ are $20,67,12.6$ and 11 respectively. 
Table 7. Comparison of the flux ratios to $\mathrm{H} \beta=100$ between the Strasbourg-ESO catalogue of galactic PN and our values for PN 1827-0729

\begin{tabular}{llrr}
\hline$\lambda(\AA)$ & Ident & Catalogue & Ours \\
\hline 4686 & HeII & & \\
4363 & {$[$ OIII $]$} & & \\
5007 & {$[\mathrm{O} I I I]$} & & 15 \\
5876 & HeI & & 61 \\
6563 & $\mathrm{H} \alpha$ & 4133 & 7044 \\
6584 & {$[\mathrm{~N}$ II] } & 4016 & 6925 \\
6717 & {$[\mathrm{SII}]$} & 139 & 192 \\
6731 & {$[\mathrm{SII}]$} & 251 & 349 \\
\hline
\end{tabular}

\section{$1827-0729$}

Kistiakowsky \& Helfland (1993) associated the radio source detected in the VLA $20 \mathrm{~cm}$ imaging survey (Zoonematkermani et al. 1990; Helfland et al. 1992) with the IRAS source, having colors typical of a PN. They imaged the PN candidate in $R$-band, $\mathrm{H} \alpha$ and [S III] $\lambda 9069$ and $\lambda 9532$. They measured an angular diameter of $2 . \prime 5 \pm 0.1$ at FWHM, which is similar to our FWHM. They didn't mention $\theta_{\mathrm{PSF}}$, but multiplying this FWHM with 1.8 would give an angular diameter of $\sim 4^{\prime \prime}$.

This object is listed in the Strasbourg-ESO catalogue of galactic PN (PNG 023.9+01.2, Acker et al. 1992; Maehara 1982), as a PN with stellar appearance, though the association with this IRAS source is not mentioned.

Our spectrum of this object shows no [O III] $\lambda 4959$ but we may observe very weak [O III] $\lambda 5007$ emission. The spectrum shows $\mathrm{H} \beta$ emission. This $\mathrm{PN}$ would be of low excitation with a stellar temperature probably around 30,000 K.

In Table 7 we compare our unreddened flux ratios to $\mathrm{H} \beta$ with those mentioned in the Strasbourg-ESO Catalogue of galactic PN. The differences are too large to be attributed to the uncertainties in our measurements. The ratio of $\mathrm{H} \alpha$ to $[\mathrm{NII}]$ and the ratio of the [SII] lines are exactly the same in both cases. Our $\mathrm{H} \alpha$ value is stronger compared to the [SII] lines, than in the catalogue. We clearly observe He I $\lambda 5876$, while it is not mentioned in the catalogue. This PN is stellar so that most of the object was in the slit. Therefore the changes in the spectrum may be real, likely due to the evolution of the central source towards higher temperature. It would be worthwile to monitor this PN.

$\underline{1839-1418}$

This $\mathrm{PN}$ has a similar morphology than $\mathrm{PN}$ $1859+1013$, though it has a more pronounced bar like structure in its center.

The spectrum of PN 1839-1418 has a higher signal-tonoise ratio than the spectrum of PN $1859+1013$. This PN also seems to have a higher excitation. It shows a strong He II line in its spectrum, but no He I emission lines were observed. The most remarkable aspect of both spectra is the absence of the $[\mathrm{N} \mathrm{II}] \lambda 6548 \& 6583$ lines, indicating a low nitrogen abundance.

\section{$1840-1109$}

The position of this $\mathrm{PN}$ is in perfect agreement with PNG 022.0-03.1 in the Catalogue of galactic PN (Acker et al. 1992), though in the catalogue the association with the IRAS source is not mentioned. Our radio flux of $36 \mathrm{mJy}$ at $6 \mathrm{~cm}$ is in agreement with the $40 \mathrm{mJy}$ at $2 \mathrm{~cm}$ measured by Milne \& Aller (1982), but much lower than the 60 mJy measured by Calabretta (1982). The optical diameter mentioned in the catalogue is $66^{\prime \prime} 4$, which is smaller than our angular size of $11^{\prime \prime}$. It is a very bright $\mathrm{PN}$ and the spectrum is the richest we obtained.

Table 8. Comparison of the flux ratios to $\mathrm{H} \beta=100$ between the Strasbourg-ESO catalogue of galactic PN and our values for PN 1840-1109

\begin{tabular}{llll}
\hline$\lambda(\AA)$ & Ident & Catalogue & Ours \\
\hline 4686 & HeII & 56 & 48 \\
4363 & {$[$ OIII $]$} & 10 & 9 \\
5007 & {$[\mathrm{O}$ III $]$} & 1435 & 1398 \\
5876 & HeI & 21 & 21 \\
6563 & H $\alpha$ & 739 & 687 \\
6584 & {$[$ NII $]$} & 76 & 87 \\
6717 & {$[\mathrm{~S}$ II $]$} & 9 & 9 \\
6731 & {$[\mathrm{~S}$ II $]$} & 14 & 14 \\
\hline
\end{tabular}

In Table 8 we compare our reddened flux ratios to $\mathrm{H} \beta$ to the ones published in the Strasbourg-ESO catalogue of galactic PN. Our values are generally less or equal than the ones published in the catalogue, though within the estimated $10 \%$ accuracy of our values, except for He II $\lambda 4686$ and $[\mathrm{N} \mathrm{II}] \lambda 6584$. The latter might be due to the deblending. In the Strasbourg-ESO catalogue the slit width was $4^{\prime \prime}$ while we used a $2^{\prime \prime}$ slit. The difference could also be due to different orientation of the slit across this extended nebula.

$1841+0343$

Lewis et al. (1987) looked for but didn't detect any OH maser emission in PN 1841+0343. The source is located in region VIII, close to region IV, in the IRAS color-color diagram (Van der Veen \& Habing 1988).

The PN is clearly bipolar and it would be interesting to investigate its morphology further.

$\underline{1858+0821}$

Only $\mathrm{H} \alpha$ and $[\mathrm{N}$ II] $\lambda 6548 \& 6583$ have been observed in this very low signal to noise spectrum. After correction for extinction the strength of the forbidden nitrogen lines relative to $\mathrm{H} \alpha=100$ are 24.4 and 66.4 respectively.

$\underline{1859+1013}$

Was identified as a new PN from the Palomar Observatory Sky Survey (POSS) plates by Capellaro et al. (1990). They mention an optical diameter of $14^{\prime \prime}$, in agreement with our measurement. 
This PN has a similar morphology than PN 1839-1418. The spectrum of PN 1859+1013 has a lower signal-to-noise ratio than the spectrum of PN 1839-1418. PN 1859+1013 also seems to be of lower excitation: it shows He I lines, but not HeII, though the latter may be caused by extinction. The most remarkable aspect of both spectra is the absence of the $[\mathrm{N}$ II $] \lambda 6548 \& 6583$ lines, indicating a low nitrogen abundance.

\section{$1908+0422$}

This spectrum was taken during the first and worst observing night. Only a few forbidden lines are clearly visible in the spectrum, confirming its PN nature. This is one of the larger, resolved $\mathrm{PN}$ of high excitation discovered, as can be deduced from its [O III] to $\mathrm{H} \alpha$ line ratio.

$\underline{1909+1326}$

This definitely seems to be a high excitation PN. It shows some [FeII], [FeIII], [FeIV], [Fe VII] emission lines in its spectrum. One would expect iron to be depleted in grains in $\mathrm{PN}$, though iron has been seen in high resolution spectra of other PN such as IC 2165 (Huyng 1994) and IC 4997 (Hyung et al. 1994). IC 2165 has a hot central star and appears to consist of zones of quite different density. IC 4997 has a variable (Hyung et al. 1994) or binary central star which is surrounded by a compact, dusty, presumably young nebula and is best fit by a thin, high density shell and a larger, lower density shell. The presence of forbidden iron emission lines probably originate in the higher density regions of these nebula. The dust temperature and dust to gas ratio is high for both nebula. In Paper I we found that PN 1909+1326 has a dust temperature of $141 \mathrm{~K}$ and a large $\mathrm{IRE}=11$.

$\underline{1911+1534}$

This PN was also observed by Garcia-Lario et al. (1990) in the $J, H$ and $K$ near IR bands $(J=12.0 \pm 0.1, H$ $=10.6 \pm 0.4, K=10.1 \pm 0.3$ ). Lewis et al. (1987) looked for but didn't detect $\mathrm{OH}$ maser emission. The IRAS source is still located in region IV of the IRAS color-color diagram as defined by Van der Veen \& Habing (1988).

The line at $\lambda=4483.6 \AA$ could be a blend of $\mathrm{Mg}$ and Fe lines (Meinel et al. 1969).

$\underline{1920+1122}$

This object doesn't seem to have a central peak, is very small and faint, however not much can be said from the image itself at this resolution.

The spectrum has low signal-to-noise, shows very weak $[\mathrm{O}$ III $] \lambda 5007$ emission and some $[\mathrm{Ar}$ III $] \lambda 7139$.

$\underline{1933-0400}$

A low resolution and low signal to noise spectrum of this $\mathrm{H} \alpha$ emission line star was published by Downes \& Keyes (1988). They did mention that superposed on a slightly red sloping continuum are low excitation forbidden lines of $[\mathrm{O} \mathrm{II}] \lambda 3727,[\mathrm{~N} \mathrm{II}] \lambda 6583$, and $[\mathrm{S} \mathrm{II}] \lambda 6717, \lambda 6730$ commonly found in PN spectra. Because no [O III] $\lambda 4959$ \& 5007 emission is observed, but the spectrum shows $\mathrm{H} \beta$, this $\mathrm{PN}$ is definitely of low excitation with a stel- lar temperature below 30,000 K. This apparently young $\mathrm{PN}$ has the lowest value for the extinction in our sample. We didn't see C IV $\lambda 4658$, which Downes \& Keyes (1988) suspected to be present, but we detected $\mathrm{C}_{\text {III }} \lambda 4648$ and Na I $\lambda 4648$ in absorption in our spectrum. The presence of He I absorption lines classify its central star as a B I spectral type.

The blue component superposed on the red continuum suggests that this object could be binary, maybe a symbiotic nebula. However the radio continuum emission argues in favor of the PN nature of this object. D-type symbiotic stars and novae also show an emission line spectrum and often have radio continuum emission above 10 mJy. However known symbiotic stars have bluer IRAS-colors than $\mathrm{PN}$. We would never have selected them as PN candidates, because their IRAS flux at $12 \mu \mathrm{m}$ is too high compared to the $25 \mu \mathrm{m}$ flux value (Whitelock 1988). It will be worthwile to keep monitoring this object.

\section{$\underline{1943+2251}$}

From the contour plot it is clear that this PN is more elongated than the three field stars around it. It would be interesting to image this $\mathrm{PN}$ at much higher resolution to investigate its morphology in more detail.

\section{Conclusions}

We presented $\mathrm{H} \alpha+[\mathrm{N}$ II] images of 17 and low resolution spectra of 14 IRAS-selected planetary nebula candidates. Almost all PN candidates selected from the IRAS PSC based on their colors typical for PN and detected in the radio continuum, were confirmed as $\mathrm{PN}$ by optical spectroscopy. Most of these IRAS PN are heavily extinct having an average $A_{V}$ of 7 mag. Large extinction may be the reason why some of the radio detected PN candidates have gone undetected in $\mathrm{H} \alpha$. On the other hand, a few IRASselected PN candidates, which were below our detection limit in the radio, have been detected in $\mathrm{H} \alpha$. About half of the PN seem to be of low excitation, having a stellar effective temperature lower than $\sim 60,000 \mathrm{~K}$. Careful modeling has been done in order to consistently determine the physical parameters of the PN with good optical spectra. The modeling used to obtain a self-consistent model and the results obtained will be discussed in forthcoming papers (Van Hoof \& Van de Steene 1996; Van de Steene \& van Hoof 1996, in preparation; Van de Steene 1995)

Acknowledgements. G.C. Van de Steene acknowledges support from the Netherlands Foundation for Research in Astronomy (ASTRON) under grant number 782-372-035. This research has made use of the Simbad database, operated at CDS, Strasbourg, France. We thank P.A.M. van Hoof for critical reading of the manuscript. 


\section{References}

Acker A., Marcout J., Ochsenbein F., Stenholm B., Tylenda R., 1992, Strasbourg-ESO Catalogue of Galactic Planetary Nebulae, ESO

Aller L.H., 1984, Physics of Thermal Gaseous Nebulae, Reidel, Dordrecht

Bedding T.R., Zijlstra A.A., 1994, A\&A 283, 955

Calabretta M.R., 1982, MNRAS 199, 141

Capellaro E., Turatto M., Salvadori L., Sabbadin F., 1990, A\&AS 86, 503

Dopita M.A., Meatheringham S.J., 1990, ApJ 357, 140

Downes R.A., Keyes C.D., 1988, AJ 96, 777

Garcia-Lario P., Manchado A., Pottasch S.R., Suso J., Olling R., 1990, A\&AS 82, 497

Helfland D.J., Zoonematkermani S., Becker R.H., White R.L., 1992, ApJS 80, 211

Hyung S., 1994, ApJS 90, 119

Huyng S., Aller L.H., Feibelman W.A., 1994, ApJS 93, 465

Iyengar K.V.K., 1987, A\&AS 68, 103

Kistiakowsky V., Helfland D.J., 1993, AJ 105, 2199

Lewis B.M., Eder J., Terzian Y., 1987, AJ 94, 1025

MacConnel D.J., 1978, A\&AS 33, 219

Maehara H., 1982, Contributions from the Bosscha Observatory No. 71

Manchado A., Garcia Lario P., Pottasch S.R., 1989, Rev. Astron. Astrofis. 18, 184

Massey P., 1992, A User's Guide to CCD Reductions with IRAF, IRAF help package
Massey P., Valdes F., Barnes J., 1992, A User's Guide to Reducing Slit Spectra with IRAF, IRAF help package

Meinel A.B., Aveni A.F., Stockton M.W., 1969, Catalog of emission lines in astrophysical objects, University of Arizona

Milne D.K., Aller L.H., 1982, A\&AS 50, 209

Osterbrock D.E., 1989, Astrophysics of Gaseous Nebulae and Active Galactic, Nuclei, University Science, California, Ch. 7

Panagia N., Walmsley C.M., 1978, A\&A 70, 411

Pottasch S.R., 1984, Planetary Nebulae, Reidel, Dordrecht, p. 93

Pottasch S.R., Bignell C., Olling R., Zijlstra A.A., 1988, A\&A 205, 248

Ratag M.A., Pottasch S.R., Zijlstra A.A., Menzies J., 1990, A\&A 233, 181

Ratag M.A., Pottasch S.R., 1991, A\&AS 91, 481

Preite-Martinez A., 1988, A\&AS 76, 317

Seaton M.J., 1979, MNRAS 187, 17

Van de Steene G.C., 1995, PhD Thesis Groningen

Van de Steene G.C., Pottasch S.R., 1993, A\&A 274, 895

Van de Steene G.C., Pottasch S.R., 1995, A\&A 299, 238 (Paper I)

Van der Veen W.E.J.C., Habing H.J., 1988, A\&A 194, 125

Whitelock P., The symbiotic phenomenon, 1987. In: Mikolajewska J., Friedjung M., Kenyon S.J., Viotti R. (eds.). Kluwer, Dordrecht

Zoonematkermani S., Helfland D.J., Becker R.H., White R.L., Perley R.A., 1990, ApJS 74, 181 


\section{A. $\mathbf{H} \alpha+[\mathrm{N} \mathrm{II}]$ images}

In this appendix all $\mathrm{H} \alpha+\left[\mathrm{N}_{\mathrm{II}}\right]$ images in which a $\mathrm{PN}$ was identified are presented as finding charts. The PN is indicated by a box. North is at the top and east is to the right. For images 1 to 7, 9 and 10, taken with the KPNO $4 \mathrm{~m}$ telescope, the field of view is $4^{\prime} \times 4^{\prime}$. For images 8 and 11 to 17 , taken wih KPNO $0.9 \mathrm{~m}$ telescope the field of view is $3^{\prime} \times 3^{\prime}$.

Fig. 1. 1818-0833
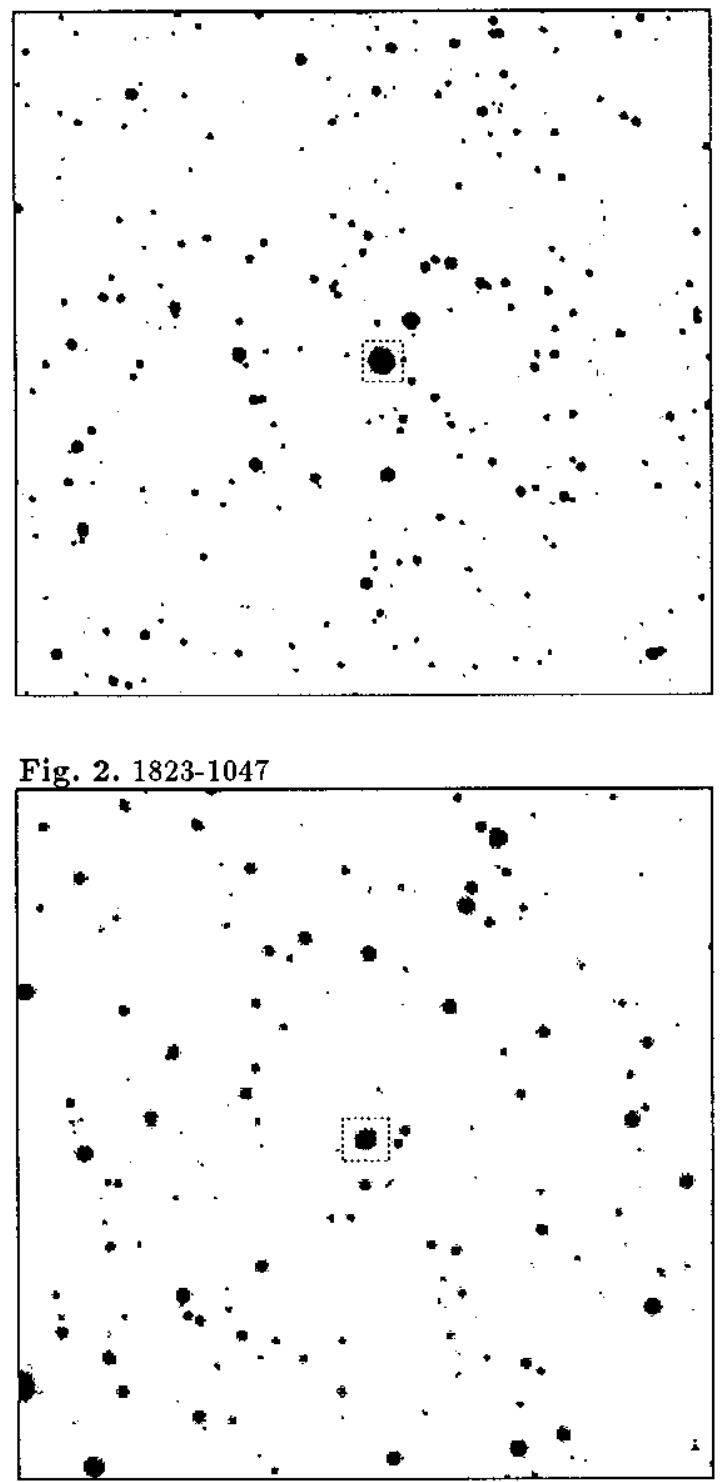

Fig. 3. 1824-1410

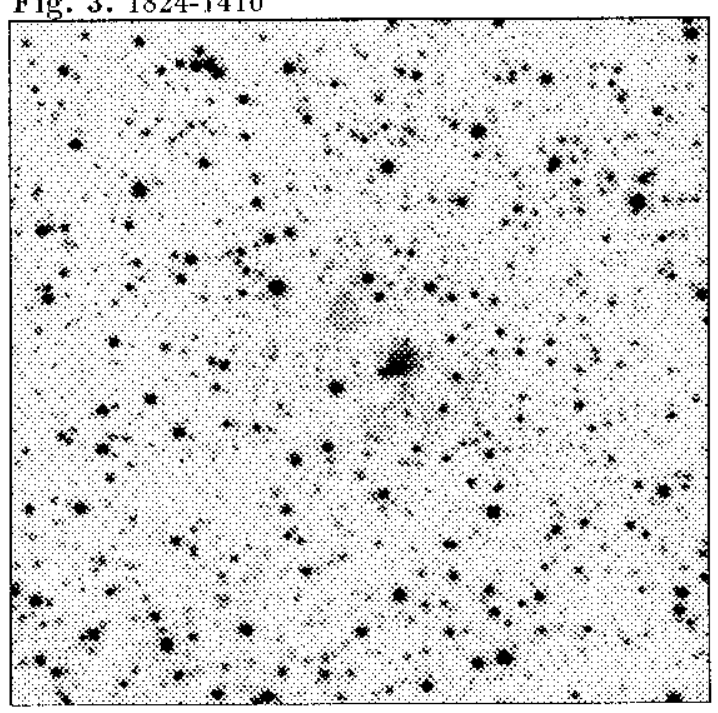

Fig. 4. 1825-0940

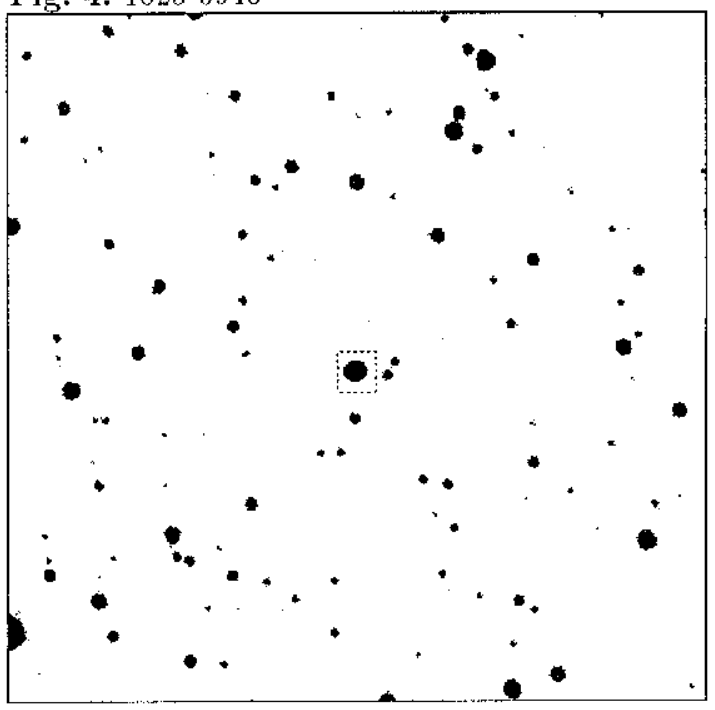

Fig. 5. 1827-0729

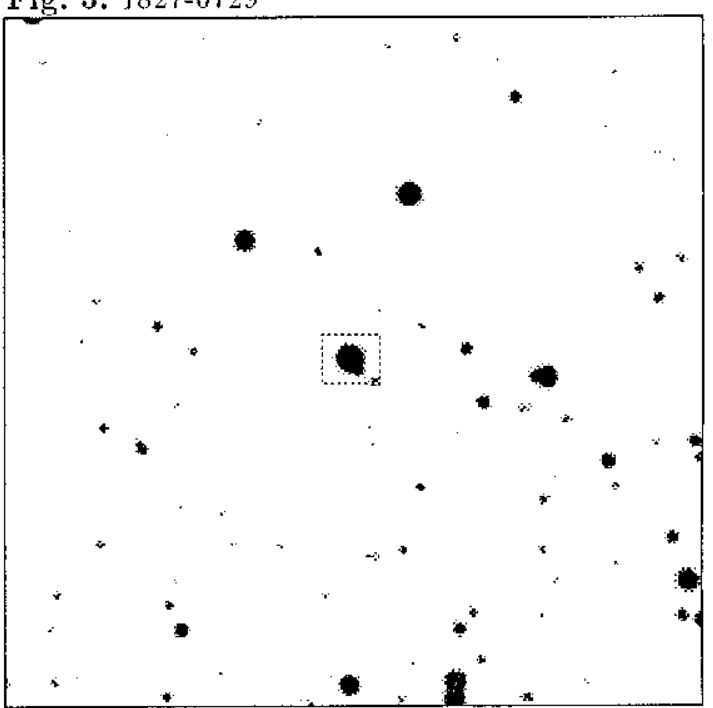


Fig. 6. 1839-1418

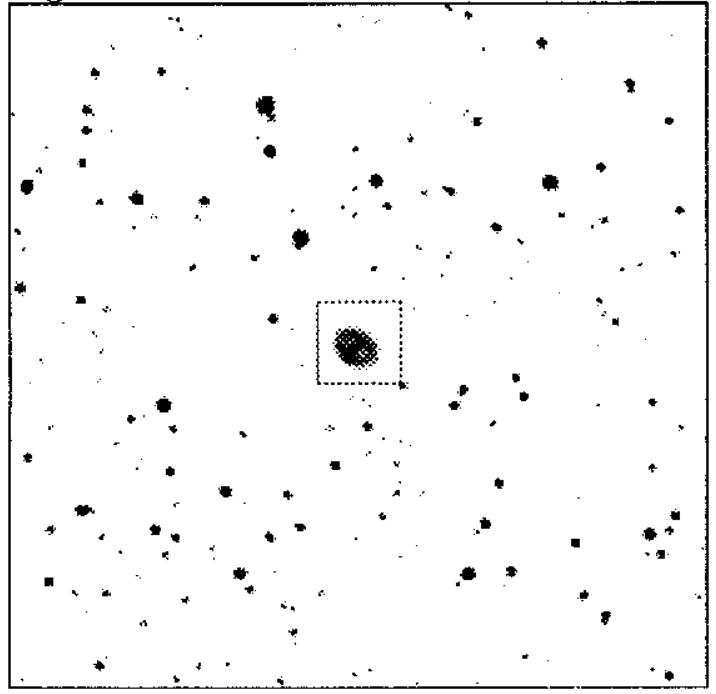

Fig. 7. 1840-1109

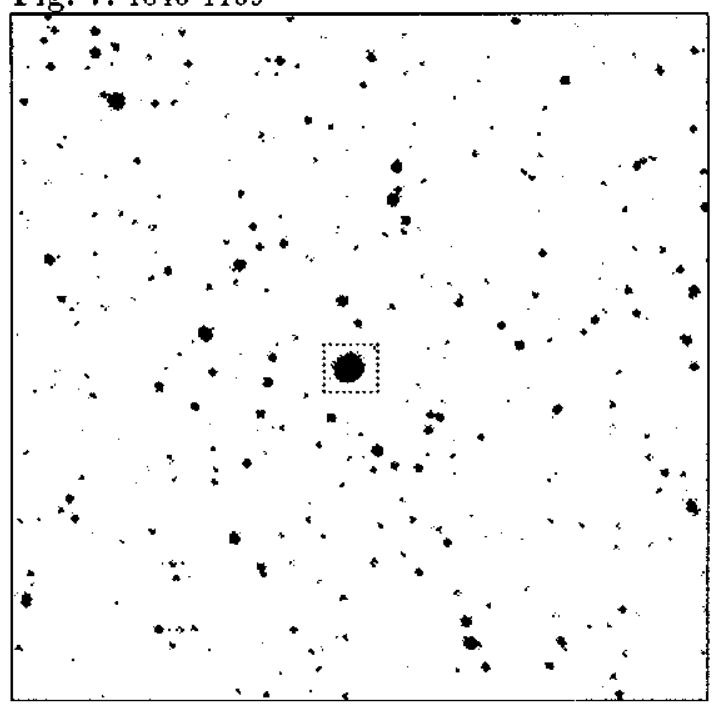

Fig. 8. $1841+0343$

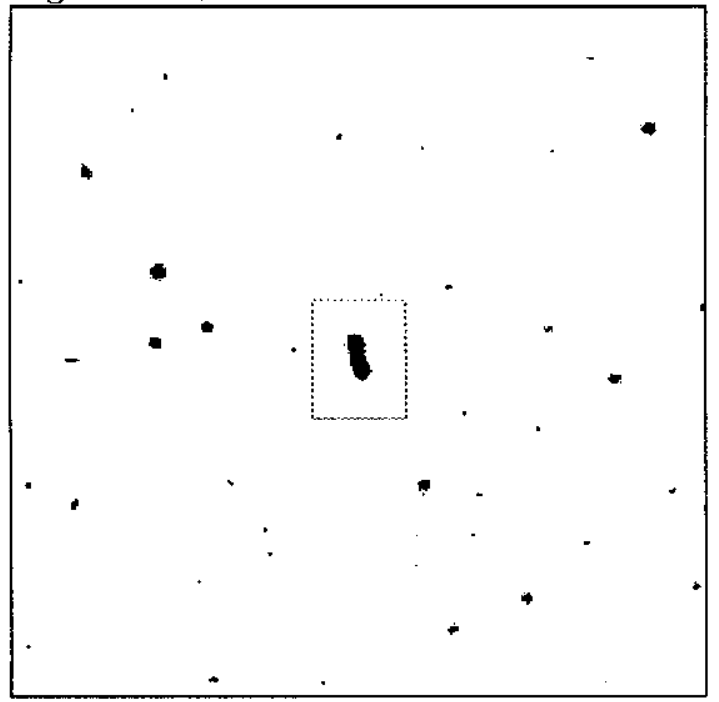

Fig. 9. $1858+0821$

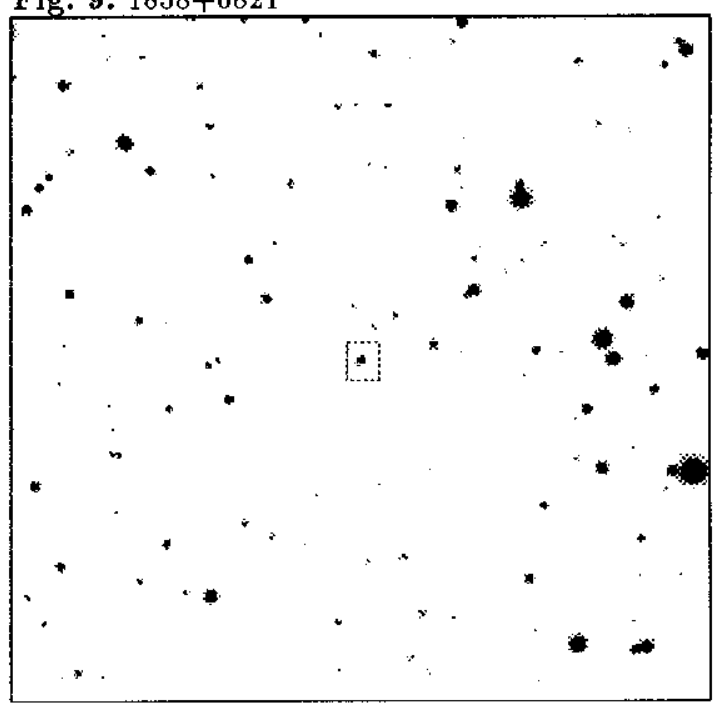

Fig. 10. $1859+1013$

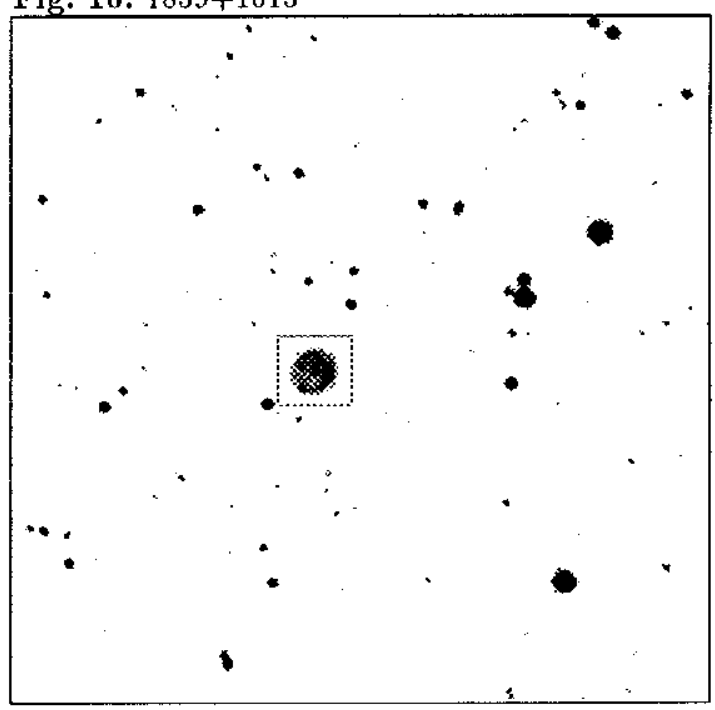

Fig. 11. $1904+1038$

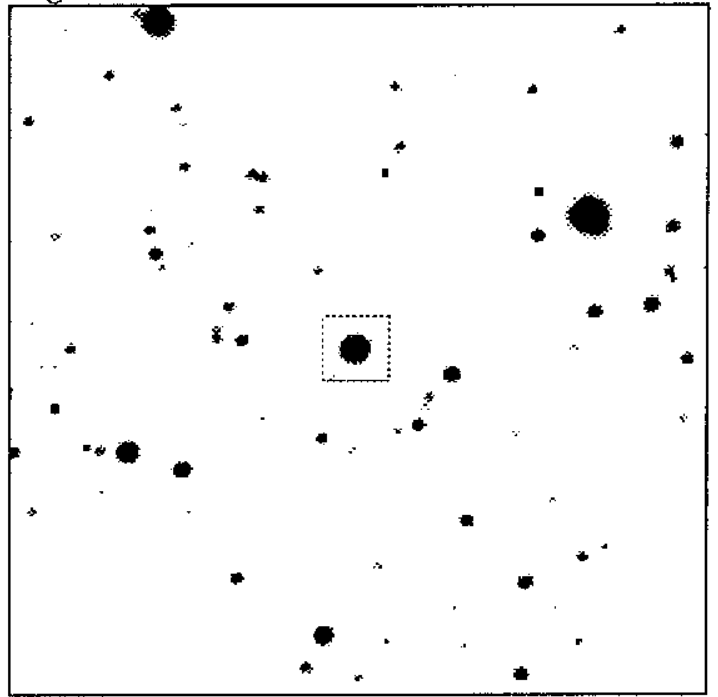


Fig. 12. $1908+0422$

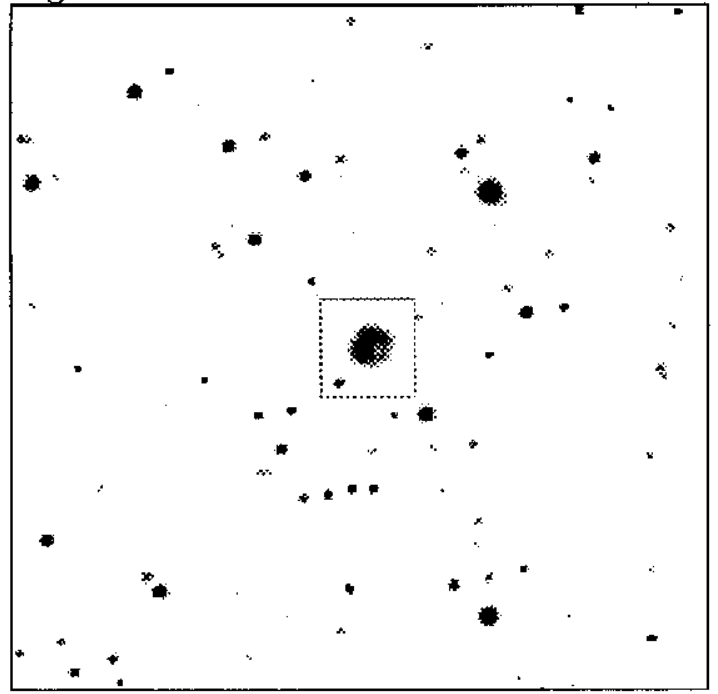

Fig. 13. $1909+1326$

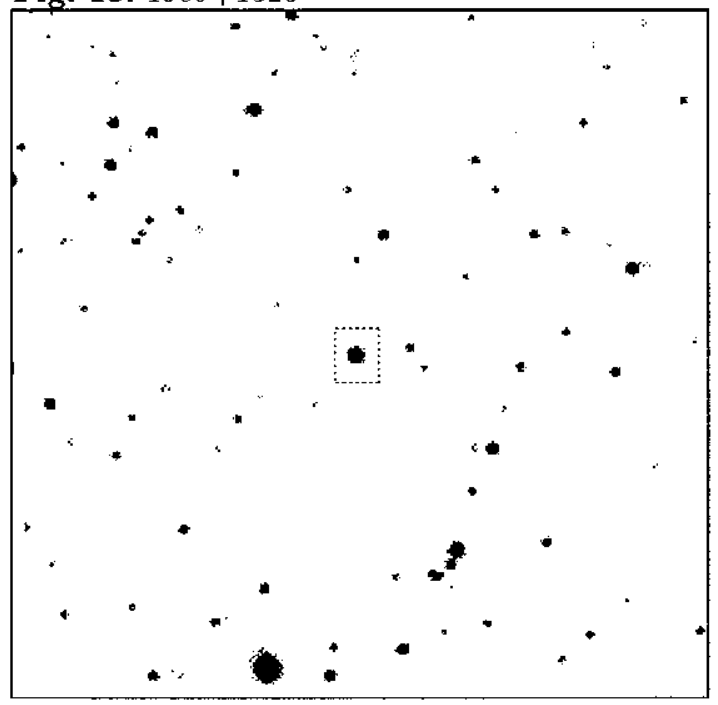

Fig. 14. $1911+1534$

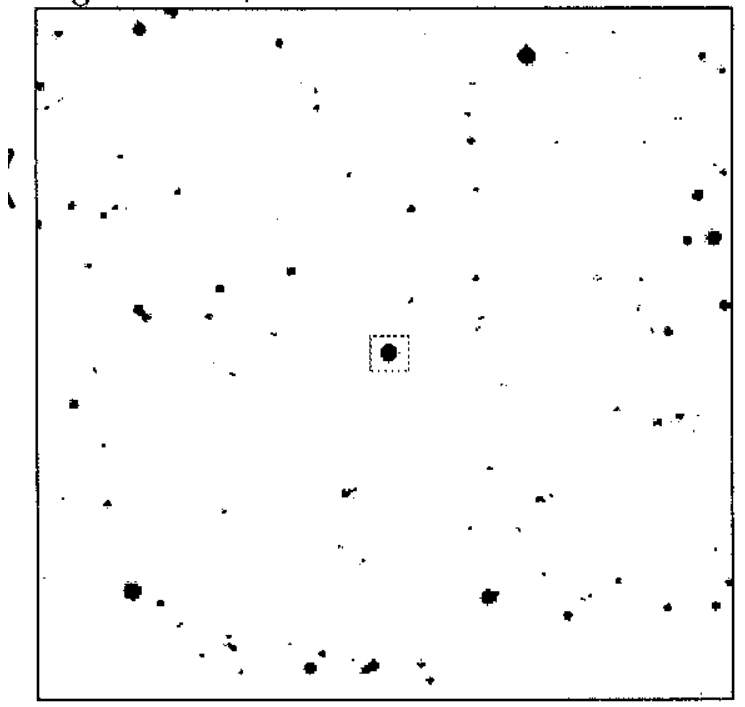

Fig. 15. $1920+1122$

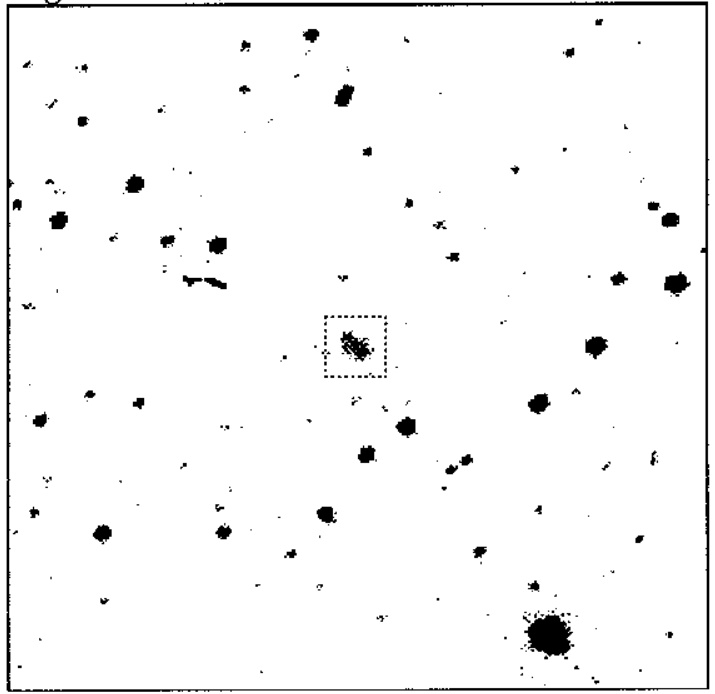

Fig. 16. 1933-0400

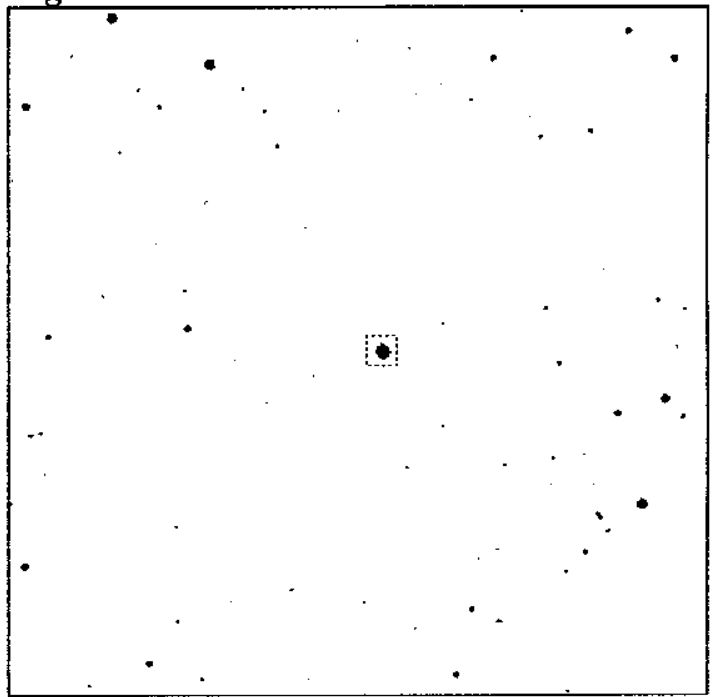

Fig. 17. $1943+2251$

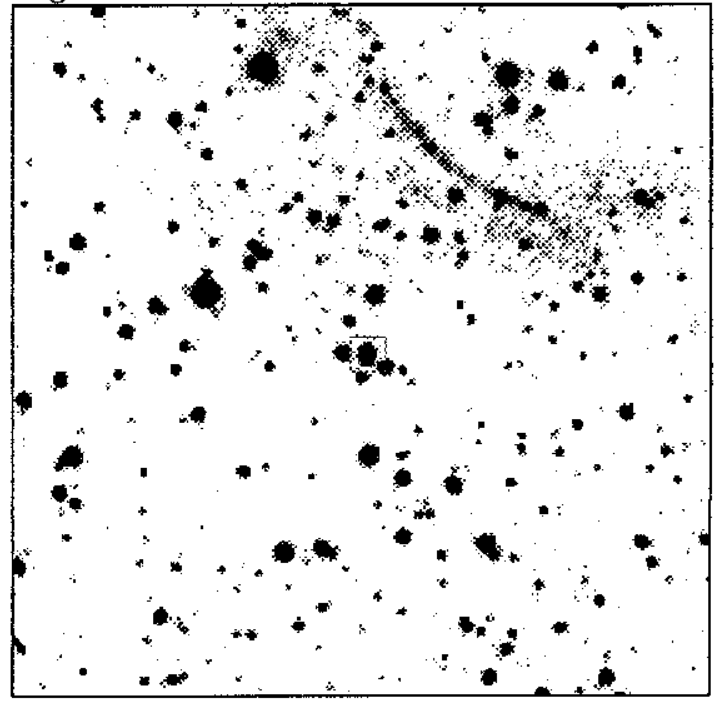




\section{B. Contour plots}
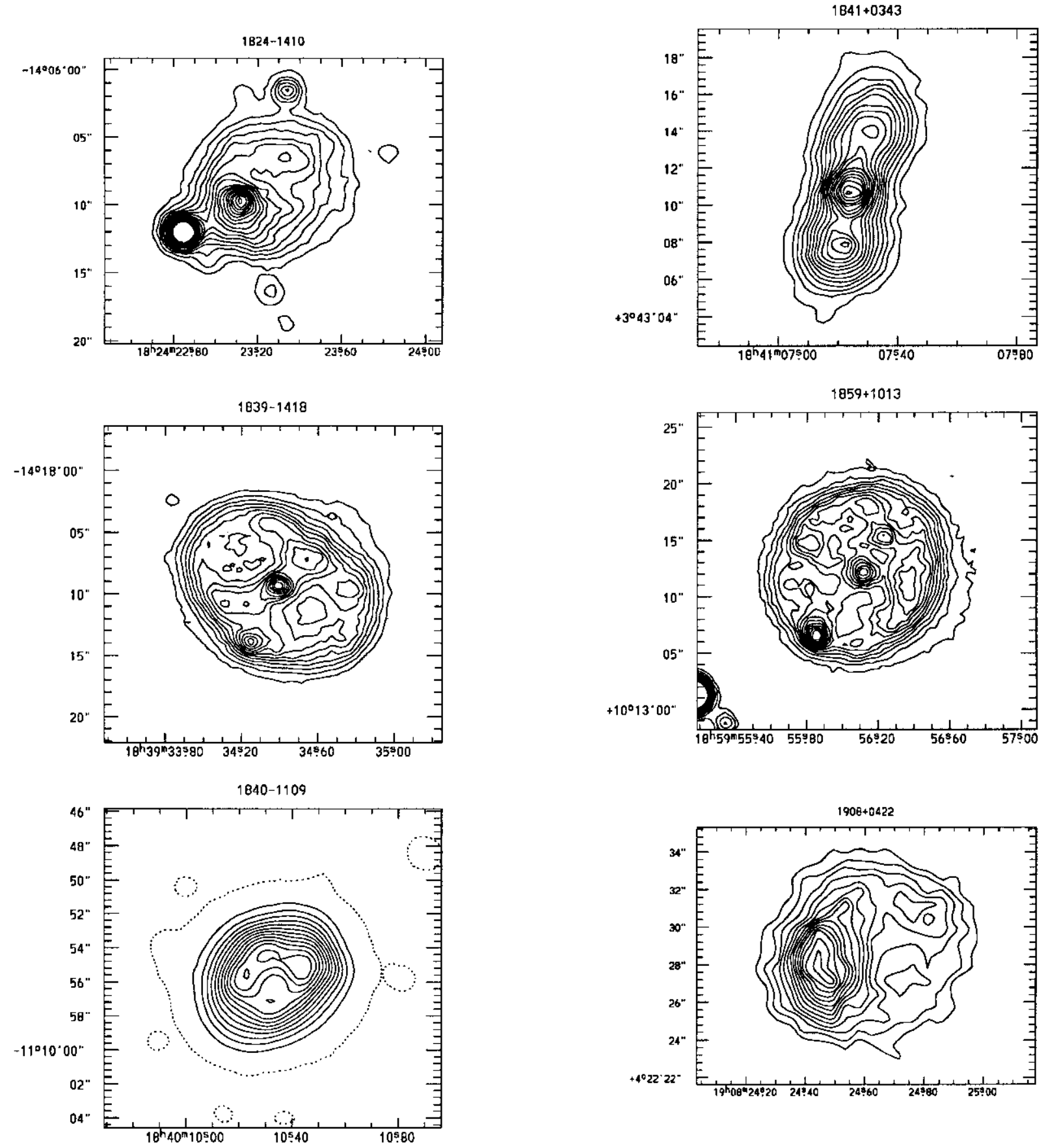

Top left: the outer, lowest contour is at $15 \%$ of the peak; the stepsize is $5 \%$ of the peak. Middle left: the outer, lowest contour is at $25 \%$ of the peak; the stepsize is $5 \%$ of the peak Bottom left: the outer, lowest contour is at $2 \%$ of the peak; the stepsize is $8 \%$ of the peak. Top right: the outer, lowest contour is at $15 \%$ of the peak; the stepsize is $5 \%$ of the peak. Middle right: the outer, lowest contour is at $35 \%$ of the peak; the stepsize is $5 \%$ of the peak Bottom right: the outer, lowest contour is at $30 \%$ of the peak; the stepsize is $5 \%$ of the peak. 

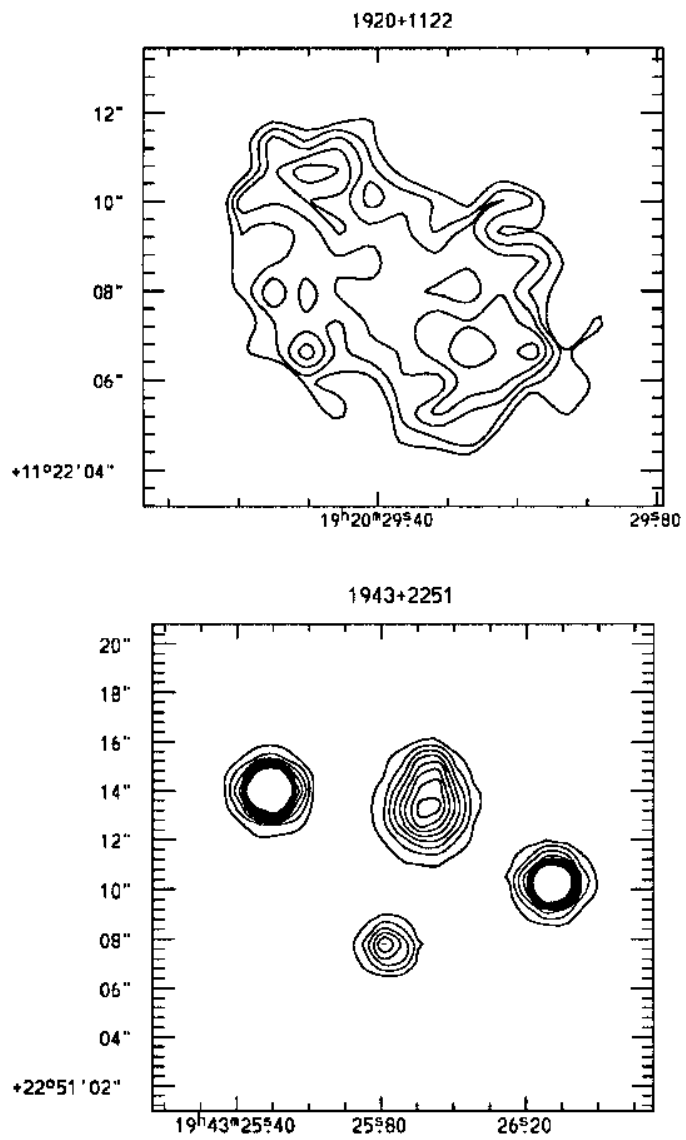

Top: the outer, lowest contour is at $75 \%$ of the peak; the stepsize is $5 \%$ of the peak.

Bottom: the outer, lowest contour is at $25 \%$ of the peak; the stepsize is $10 \%$ of the peak. 
C. Optical spectra
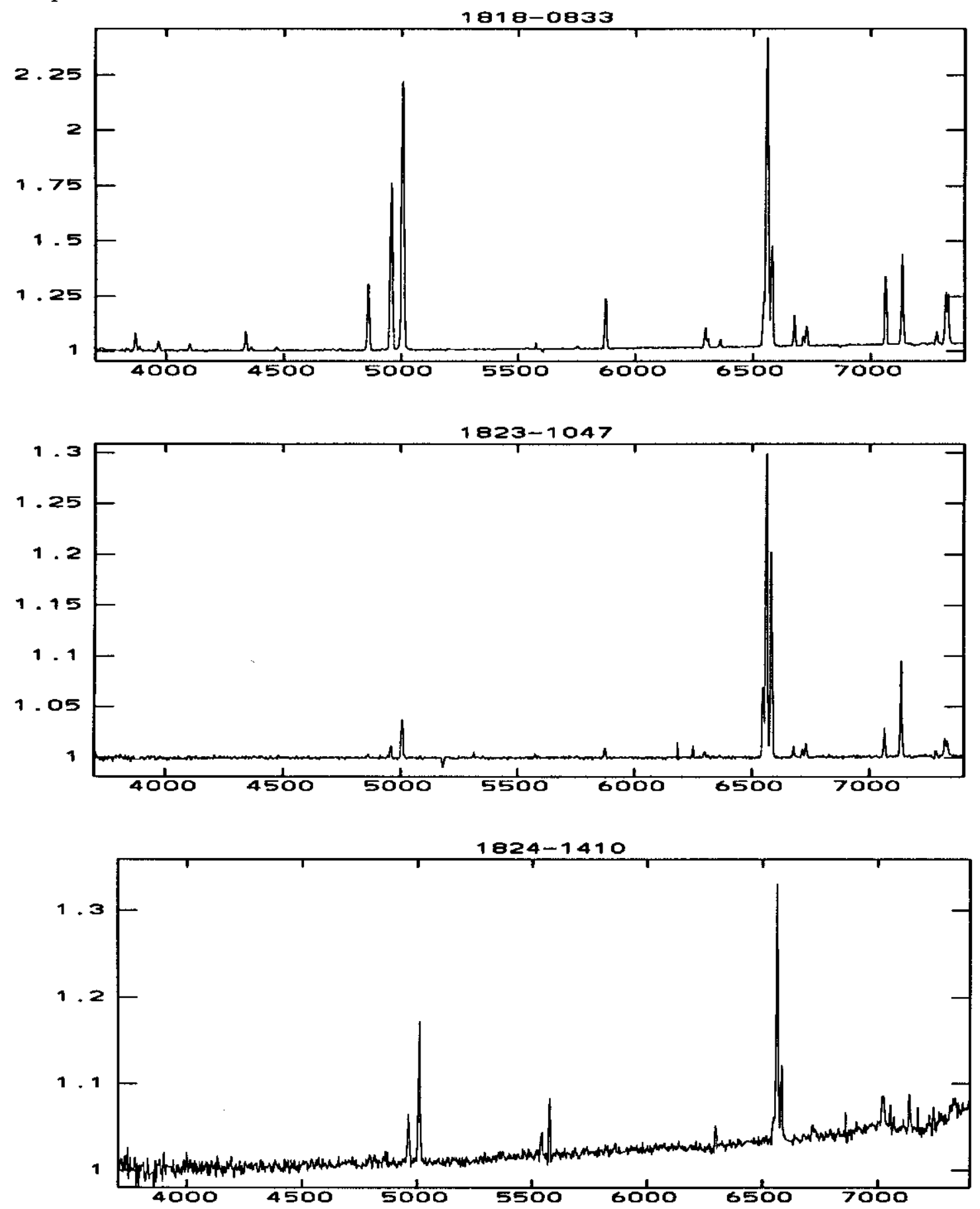

Spectra of IRAS-selected planetary nebulae. Above every spectrum the object is mentioned.

$X$-axis: wavelength from $3.700 \AA$ to $7.400 \AA$

$Y$-axis: $\log \left(10+I 10^{15}\right)$ in $\operatorname{ergs} / \mathrm{cm}^{2} / \mathrm{s} / \AA$. 

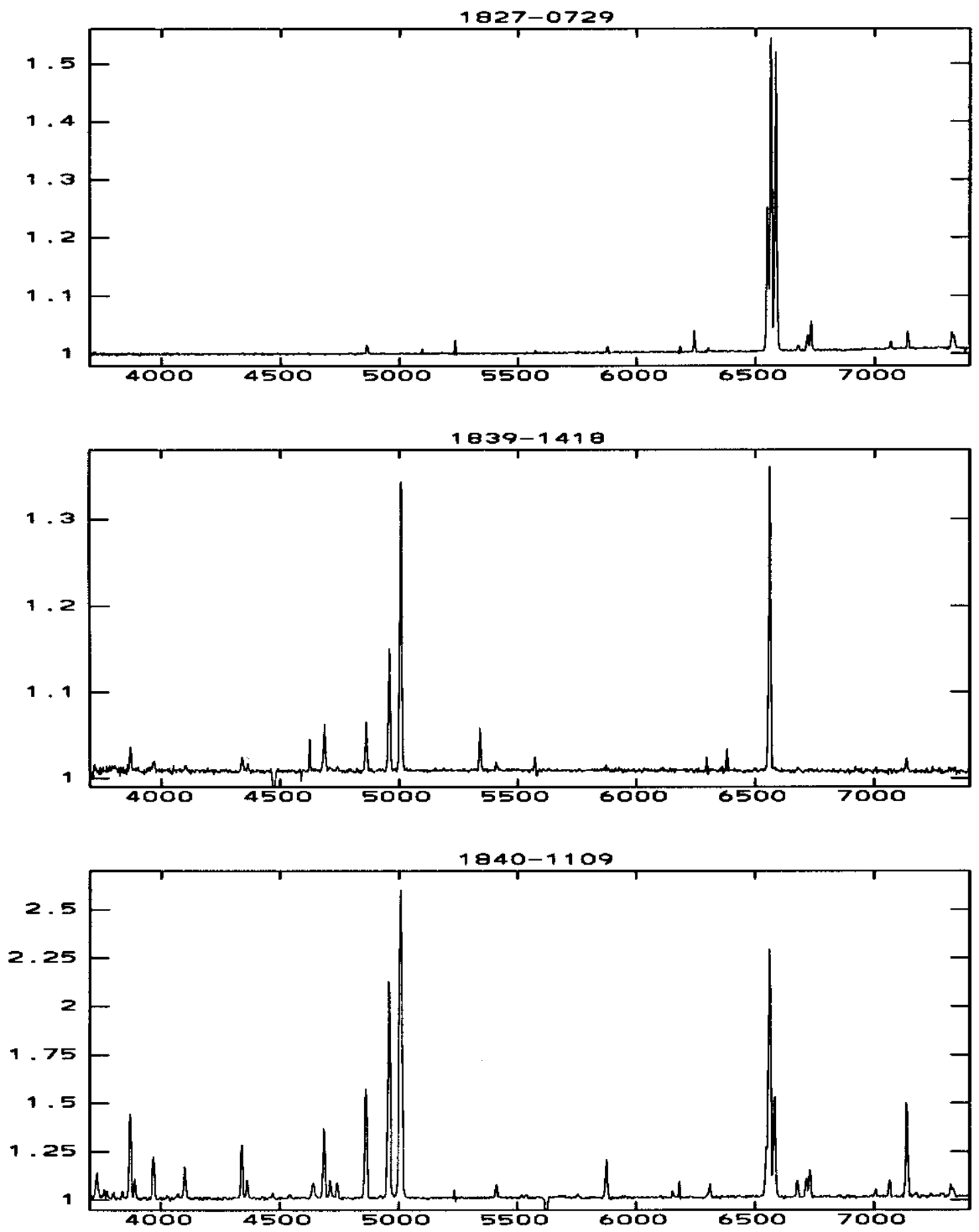

Spectra of IRAS-selected planetary nebulae. Above every spectrum the object is mentioned.

$X$-axis: wavelength from $3.700 \AA$ to $7.400 \AA$

$Y$-axis: $\log \left(10+I 10^{15}\right)$ in $\operatorname{ergs} / \mathrm{cm}^{2} / \mathrm{s} / \AA$. 

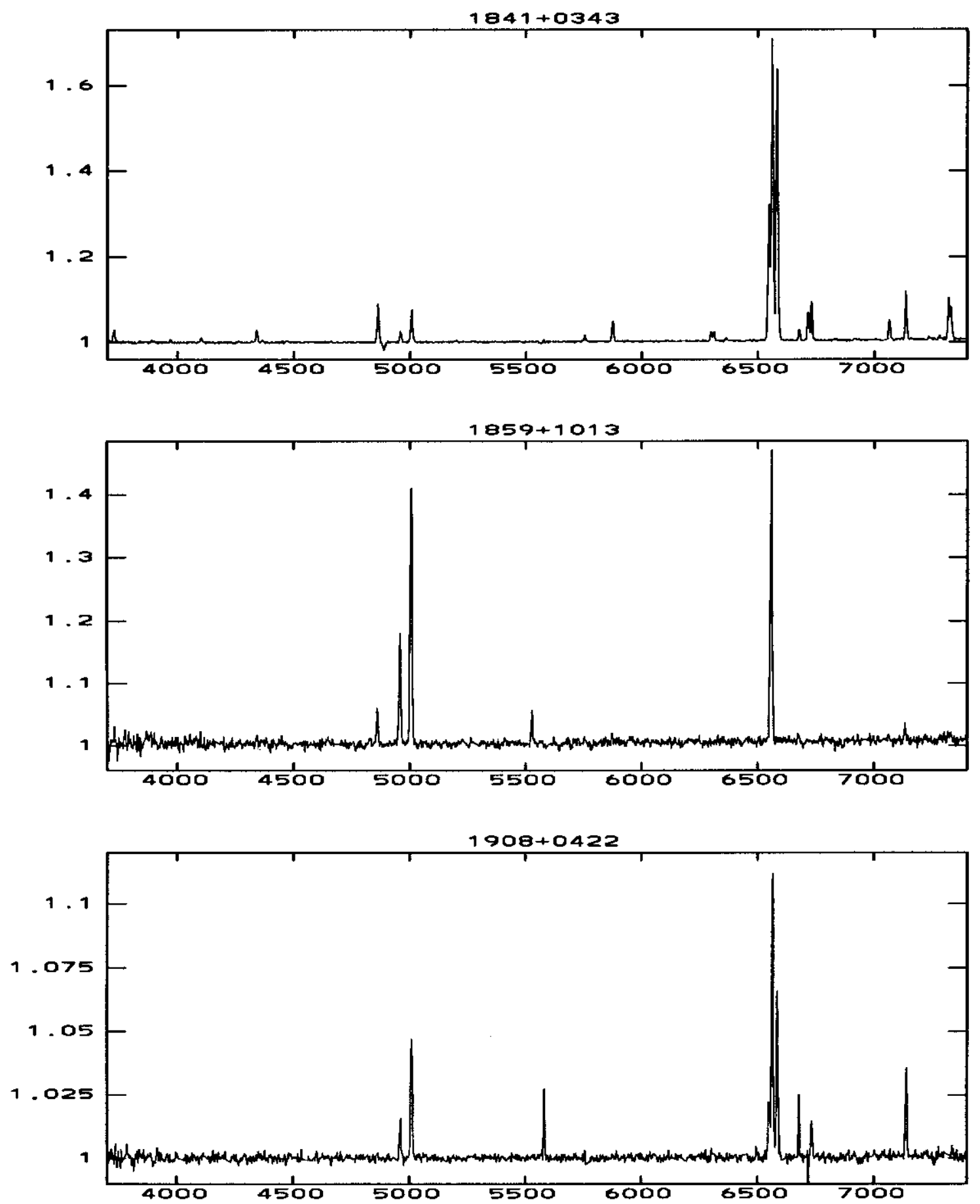

Spectra of IRAS-selected planetary nebulae. Above every spectrum the object is mentioned.

$X$-axis: wavelength from $3.700 \AA$ to $7.400 \AA$

$Y$-axis: $\log \left(10+I 10^{15}\right)$ in $\operatorname{ergs} / \mathrm{cm}^{2} / \mathrm{s} / \AA$. 

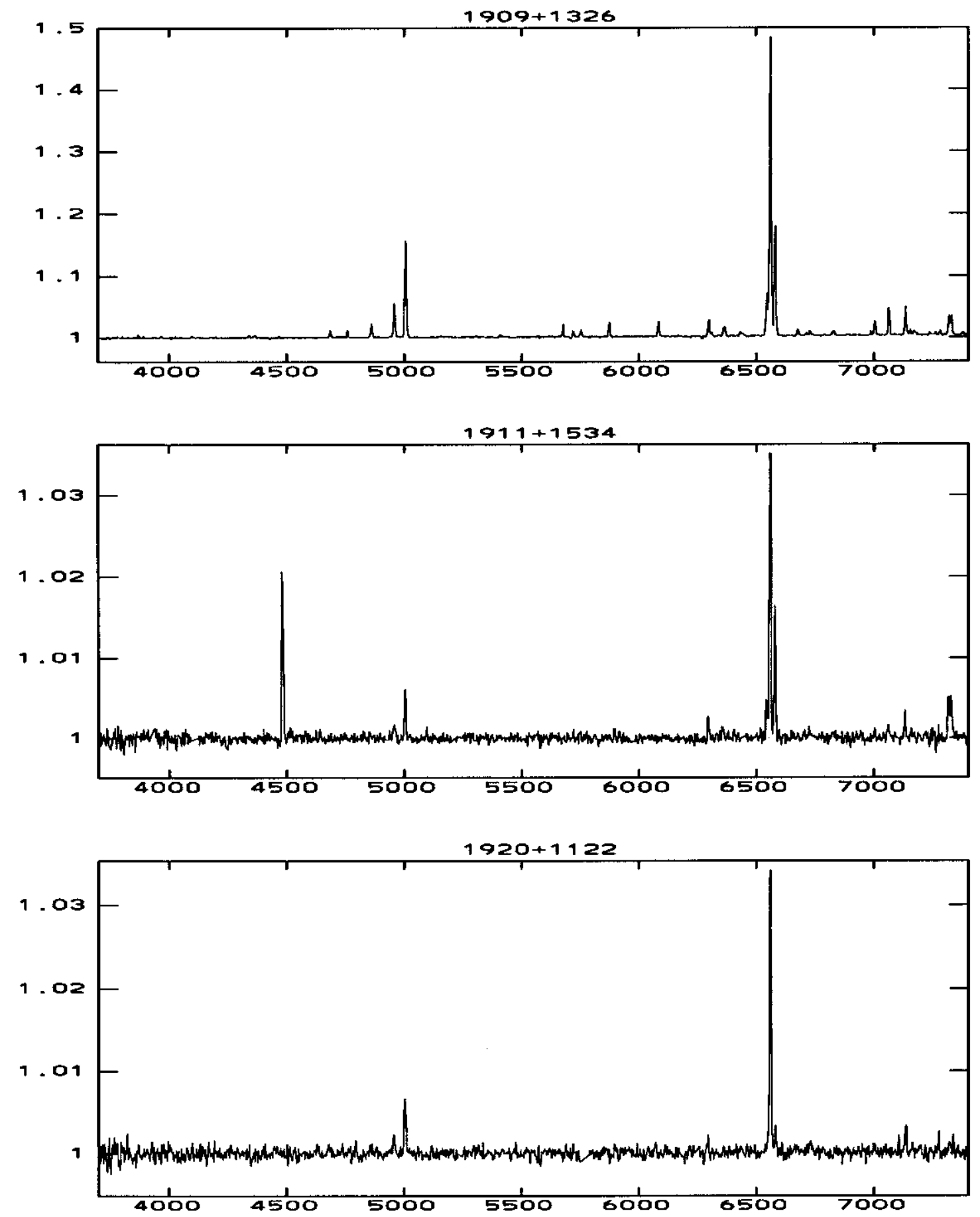

Spectra of IRAS-selected planetary nebulae. Above every spectrum the object is mentioned.

$X$-axis: wavelength from $3.700 \AA$ to $7.400 \AA$

$Y$-axis: $\log \left(10+I 10^{15}\right)$ in $\operatorname{ergs} / \mathrm{cm}^{2} / \mathrm{s} / \AA$. 

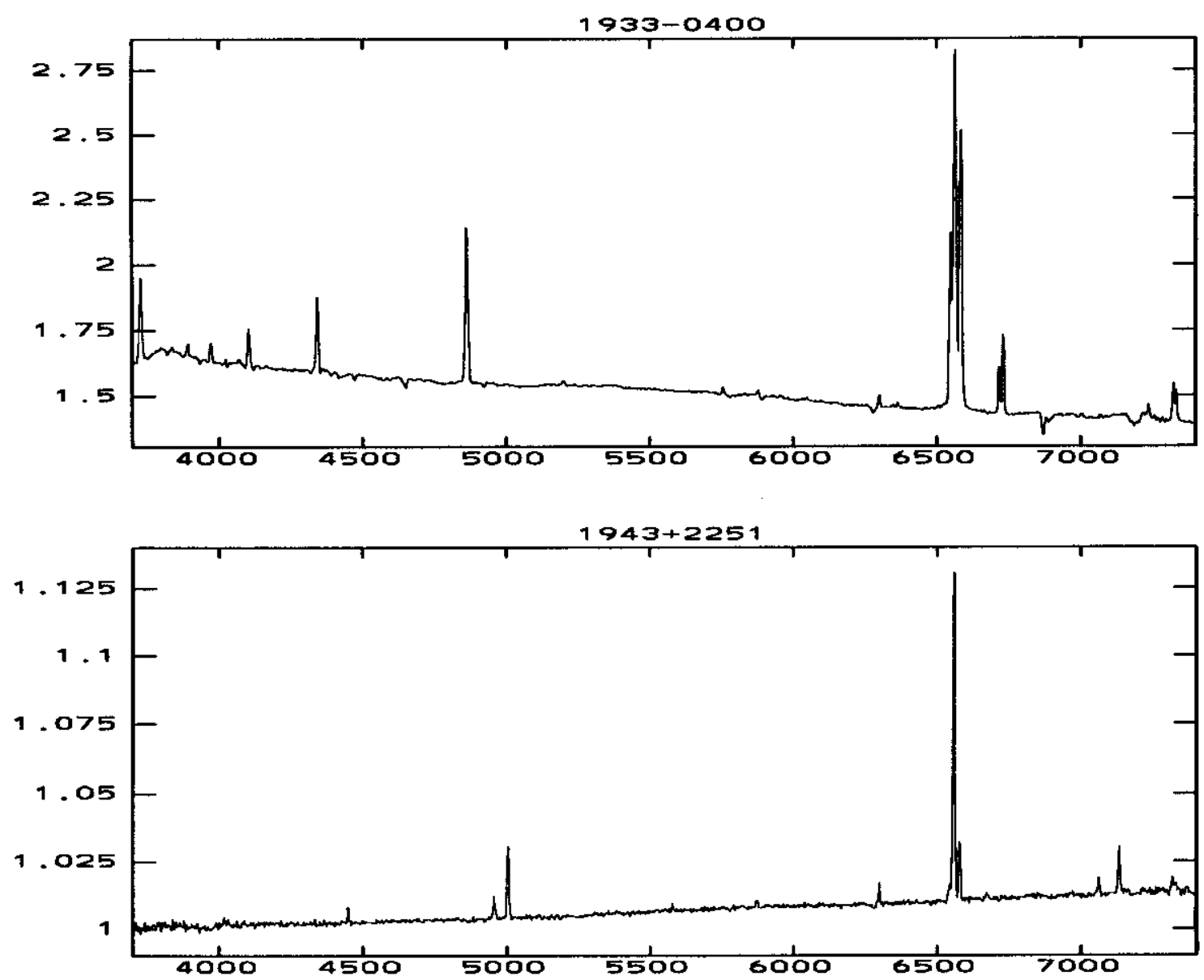

Spectra of IRAS-selected planetary nebulae. Above every spectrum the object is mentioned.

$X$-axis: wavelength from $3.700 \AA$ to $7.400 \AA$

$Y$-axis: $\log \left(10+I 10^{15}\right)$ in $\mathrm{ergs} / \mathrm{cm}^{2} / \mathrm{s} / \AA$. 


\section{Line flux ratios}

Line fluxes corrected for interstellar extinction relative to $\mathrm{H} \beta$ or [O III] $\lambda 5007$.

An estimate of the percentual uncertainty in the relative flux (err) is also given.

Contaminated lines are indicated by a colon.

\begin{tabular}{|c|c|c|c|c|c|c|c|c|c|c|c|c|c|c|c|}
\hline \multirow[t]{2}{*}{$\overline{\lambda(\AA)}$} & \multirow[t]{2}{*}{ Ident } & \multicolumn{2}{|c|}{$1818-0833$} & \multicolumn{2}{|c|}{$1823-1047$} & \multicolumn{2}{|c|}{$1824-1410$} & \multicolumn{2}{|c|}{$1827-0729$} & \multicolumn{2}{|c|}{$1839-1418$} & \multicolumn{2}{|c|}{$1840-1109$} & \multicolumn{2}{|c|}{$1841+0343$} \\
\hline & & Flux & err & Flux & err & Flux & err & Flux & err & Flux & err & Flux & $\overline{\text { err }}$ & Flux & err \\
\hline$\overline{3727}$ & [O II] & & & & & & & & & & & 28.3 & 10 & 138.6 & 10 \\
\hline 3750 & $\mathrm{H} \mathrm{I}$ & & & & & & & & & & & & & & \\
\hline 3771 & H I & & & & & & & & & & & 3.1 & 15 & & \\
\hline 3798 & H I & & & & & & & & & & & & & & \\
\hline 3835 & H I & & & & & & & & & & & 4.9 & 10 & & \\
\hline 3869 & [Ne III] & 88.0 & 10 & & & & & & & 92.9 & 10 & 110.3 & 10 & & \\
\hline 3889 & $\mathrm{He} \mathrm{I}, \mathrm{H} \mathrm{I}$ & 21.1 & 12 & & & & & & & & & 16.6 & 10 & 5.9 & 57 \\
\hline 4026 & $\mathrm{He} \mathrm{I}$ & 1.9 & 46 & & & & & & & & & 1.1: & 36 & & \\
\hline 4069 & [S II] & & & & & & & & & & & 1.4 & 28 & & \\
\hline 4076 & {$[\mathrm{~S} \mathrm{II}]$} & & & & & & & & & & & & & & \\
\hline 4102 & $\mathrm{H} \mathrm{I}$ & 20.2 & 10 & & & & & & & 22.0 & 19 & 25.9 & 10 & 20.8 & 13 \\
\hline 4340 & H I & 47.4 & 10 & & & & & & & 50.7 & 10 & 45.9 & 10 & 56.1 & 10 \\
\hline 4363 & [O III] & 7.0 & 10 & & & & & & & 19.7 & 16 & 11.7 & 10 & & \\
\hline 4471 & $\mathrm{He} \mathrm{I}$ & 6.8 & 17 & & & & & & & & & 2.6 & 13 & & \\
\hline 4686 & He II & & & & & & & & & 99.1 & 10 & 53.3 & 10 & & \\
\hline 4740 & [Ar IV] & 2.1 & 16 & & & & & & & 4.9 & 43 & 7.5 & 10 & & \\
\hline 4861 & H I & 100.0 & 10 & 11.1 & 28 & 8.9 & 18 & 100.0 & 10 & 100.0 & 10 & 100.0 & 10 & 100.0 & 10 \\
\hline 4959 & [O III] & 403.0 & 10 & 31.8 & 10 & 36.6 & 10 & & & 251.1 & 10 & 423.1 & 10 & 21.9 & 10 \\
\hline 5007 & [O III] & 1217 & 10 & 100.0 & 10 & 100.0 & 10 & 10.0 & 50 & 754.1 & 10 & 1266 & 10 & 69.6 & 10 \\
\hline 5309 & {$[\mathrm{Ca} \mathrm{V}]$} & & & 3.7 & 20 & & & & & & & & & & \\
\hline 5518 & [Cl III] & & & & & & & & & & & 1.3 & 22 & & \\
\hline 5538 & [Cl III] & 0.6 & 21 & & & & & & & & & 1.7 & 35 & & \\
\hline 5577 & [O I] & & & 0.8 & 51 & 11.3 & 10 & & & 7.6 & 15 & & & 1.4: & 30 \\
\hline 5755 & [N II] & 0.8 & 13 & 0.5 & 49 & & & 1.4 & 50 & & & 0.5 : & 25 & 3.1 & 10 \\
\hline 5876 & $\mathrm{He} \mathrm{I}$ & 16.5 & 10 & 1.9 & 10 & & & 7.2 & 10 & & & 11.6 & 10 & 15.4 & 10 \\
\hline 6300 & [O I] & 3.4 & 10 & 0.5 & 15 & 1.8 & 11 & 1.4 & 16 & 4.3 & 17 & 0.7 & 22 & 4.6 & 10 \\
\hline 6312 & [S III] & 1.3 & 10 & & & & & 0.6 & 43 & & & 3.1 & 10 & 4.3 & 10 \\
\hline 6364 & [O I] & 1.1 & 10 & 0.1 & 53 & & & & & & & & & $0.5:$ & 41 \\
\hline 6435 & {$[\mathrm{Ar} \mathrm{V}]$} & & & & & & & & & & & & & & \\
\hline 6548 & [N II] & 8.4 & 10 & 5.7 & 10 & 2.7 & 26 & 89.3 & 10 & & & 13.0 & 10 & 78.0 & 10 \\
\hline 6563 & H I & 285.0 & 10 & 31.8 & 10 & 25.2 & 10 & 285.0 & 10 & 285.0 & 10 & 285.0 & 10 & 285.0 & 10 \\
\hline 6583 & [N II] & 19.8 & 10 & 17.6 & 10 & 4.9 & 10 & 270.1 & 10 & & & 36.3 & 10 & 245.1 & 10 \\
\hline 6678 & $\mathrm{HeI}$ & 4.0 & 10 & 0.5 & 10 & & & 1.8 & 10 & & & 3.4 & 10 & 4.0 & 10 \\
\hline 6716 & [S II] & 1.3 & 10 & 0.5 & 10 & 1.4 & 22 & 6.2 & 10 & & & 3.6 & 10 & 10.0 & 10 \\
\hline 6731 & [S II] & 2.5 & 10 & 0.9 & 10 & & & 11.2 & 10 & & & 5.6 & 10 & 14.7 & 10 \\
\hline 7006 & {$[\mathrm{Ar} \mathrm{V}]$} & & & & & & & & & & & 1.1 & 10 & & 10 \\
\hline 7065 & $\mathrm{He} \mathrm{I}$ & 8.9 & 10 & 1.0 & 10 & & & 1.5 & 10 & & & 3.4 & 10 & 4.8 & 10 \\
\hline 7136 & [Ar III] & 12.3 & 10 & 3.3 & 10 & 1.8 & 10 & 1.8 & 10 & 7.5 & 10 & 27.4 & 10 & 14.0 & 10 \\
\hline 7281 & $\mathrm{He} \mathrm{I}$ & 1.0 & 10 & 0.1 & 13 & & & 0.5 & 18 & & & 0.4 & 21 & 0.4 & 33 \\
\hline 7320 & [O II] & 4.6 & 10 & 0.4 & 10 & 1.2 & 24 & 3.1 & 10 & 2.1 & 20 & 1.9 & 10 & 10.3 & 10 \\
\hline 7331 & {$[\mathrm{O}$ II $],[\mathrm{Ar}$ IV $]$} & 4.7 & 10 & 0.3 & 10 & 0.8 & 12 & 2.9 & 10 & 2.3 & 24 & 1.6 & 10 & 8.9 & 10 \\
\hline
\end{tabular}


Line fluxes corrected for interstellar extinction relative to $\mathrm{H} \beta$ or $[\mathrm{O}$ III $] \lambda 5007$.

An estimate of the percentual uncertainty in the relative flux (err) is also given.

Contaminated lines are indicated by a colon.

\begin{tabular}{|c|c|c|c|c|c|c|c|c|c|c|c|c|c|c|c|}
\hline$\lambda(\AA)$ & Ident & $1859+$ & 013 & $1908+$ & 422 & $1909+$ & 326 & $1911 t$ & 534 & 19204 & 122 & 1933-c & & $1943+$ & 251 \\
\hline & & Flux & err & Flux & err & Flux & err & Flux & err & Flux & err & Flux & err & Flux & err \\
\hline$\overline{3727}$ & {$[\mathrm{O} \mathrm{II}]$} & & & & & & & & & & & 69.3 & 10 & & \\
\hline 3750 & H I & & & & & & & & & & & & & & \\
\hline 3771 & H I & & & & & & & & & & & & & & \\
\hline 3798 & H I & & & & & & & & & & & & & & \\
\hline 3835 & $\mathrm{HI}$ & & & & & & & & & & & 1.5 & 10 & & \\
\hline 3869 & [Ne III] & & & & & 1.6 & 40 & & & & & & & & \\
\hline 3889 & He I, H I & & & & & 5.7 & 75 & & & & & 5.5 & 10 & & \\
\hline 4026 & $\mathrm{HeI}$ & & & & & & & & & & & & & & \\
\hline 4069 & [S II] & & & & & & & & & & & 1.8 & 10 & & \\
\hline 4076 & [S II] & & & & & & & & & & & & & & \\
\hline 4102 & $\mathrm{H} \mathrm{I}$ & & & & & & & & & & & 22.4 & 10 & & \\
\hline 4340 & $\mathrm{HI}$ & & & & & 35.2 & 11 & & & & & 45.2 & 10 & & \\
\hline 4363 & [O III] & & & & & 42.4 & 11 & & & & & & & & \\
\hline 4471 & $\mathrm{He}$ I & & & & & 2.5 & 90 & & & & & & & & \\
\hline 4686 & He II & & & & & 61.2 & 10 & & & & & & & & \\
\hline 4740 & [Ar IV] & & & & & 3.6 & 57 & & & & & & & & \\
\hline 4861 & H I & 100.0 & 10 & & & 100.0 & 10 & & & & & 100.0 & 10 & & \\
\hline 4959 & [O III] & 293.6 & 10 & 24.1 & 10 & 203.4 & 10 & 12.1 & 51 & 30.5 & 23 & & & 34.8 & 10 \\
\hline 5007 & [O III] & 884.4 & 10 & 100.0 & 10 & 604.9 & 10 & 100.0 & 10 & 100.0 & 10 & & & 100.0 & 10 \\
\hline 5309 & {$[\mathrm{Ca} \mathrm{V}]$} & & & & & & & & & & & & & & \\
\hline 5518 & [Cl III] & & & & & & & & & & & & & & \\
\hline 5538 & [Cl III] & & & & & & & & & & & & & & \\
\hline 5577 & [O I] & & & & & & & & & & & & & 1.7 & 24 \\
\hline 5755 & [N II] & 7.0 & 48 & & & 11.2 & 10 & & & & & 1.3 & 10 & & \\
\hline 5876 & $\mathrm{He} \mathrm{I}$ & 5.7 & 34 & & & 15.5 & 10 & & & & & 0.7 & 10 & 1.5 & 25 \\
\hline 6300 & [O I] & & & & & 12.6 & 10 & 4.9 & 13 & & & 1.7 & 10 & 1.3 & 10 \\
\hline 6312 & [S III] & & & & & 2.9 & 10 & & & & & & & & \\
\hline 6364 & {$[\mathrm{OI}]$} & & & & & 4.3 & 10 & & & & & & & & \\
\hline 6435 & {$[\mathrm{Ar} \mathrm{V}]$} & & & & & 3.8 & 26 & & & & & & & & \\
\hline 6548 & {$[\mathrm{~N} \mathrm{III}]$} & & & 2.5 & 10 & 27.3 & 10 & 7.2 & 10 & & & 45.8 & 10 & 1.4 & 10 \\
\hline 6563 & H I & 285.0 & 10 & 16.1 & 10 & 285.0 & 10 & 50.4 & 10 & 33.9 & 1 & 285.0 & 10 & 26.5 & 10 \\
\hline 6583 & {$\left[\begin{array}{lll}\mathrm{N} & 11\end{array}\right]$} & & & 8.0 & 10 & 69.8 & 10 & 21.2 & 10 & 1.8 & 19 & 137.4 & 10 & 3.9 & 10 \\
\hline 6678 & $\mathrm{HeI}$ & & & & & 3.3 & 10 & & & & & & & 0.4 & 25 \\
\hline 6716 & [S II] & & & 1.5 & 10 & 1.1 & 11 & & & & & 5.7 & 10 & & \\
\hline 6731 & [S II] & & & 1.6 & 10 & & & & & & & 11.2 & 10 & & \\
\hline 7006 & {$[\mathrm{ArV}]$} & & & & & 4.9 & 10 & & & & & & & & \\
\hline 7065 & $\mathrm{He} \mathrm{I}$ & 2.1 & 43 & & & 9.1 & 10 & & & & & & & 0.7 & 10 \\
\hline 7136 & [Ar III] & 6.0 & 17 & 2.1 & 10 & & & 2.4 & 11 & 1.6 & 15 & & & 1.6 & 10 \\
\hline 7281 & $\mathrm{HeI}$ & & & & & 1.1 & 10 & & & & & & & & \\
\hline 7320 & [O II] & & & & & 5.4 & 10 & 2.4 & 10 & & & 3.5 & 10 & 0.2 & 15 \\
\hline 7331 & [O II], [Ar IV] & & & & & 5.2 & 10 & 3.2 & 10 & & & 3.1 & 10 & 0.2 & 45 \\
\hline
\end{tabular}

